\title{
The Stress Protein/Chaperone Grp94 Counteracts Muscle Disuse Atrophy by Stabilizing Subsarcolemmal Neuronal Nitric Oxide Synthase
}

\author{
Maurizio Vitadello, Jennifer Gherardini, ${ }^{1}$ and Luisa Gorza ${ }^{2}$
}

\begin{abstract}
Aims: Redox and growth-factor imbalance fosters muscle disuse atrophy. Since the endoplasmic-reticulum chaperone Grp94 is required for folding insulin-like growth factors (IGFs) and for antioxidant cytoprotection, we investigated its involvement in muscle mass loss due to inactivity. Results: Rat soleus muscles were transfected in vivo and analyzed after 7 days of hindlimb unloading, an experimental model of muscle disuse atrophy, or standard caging. Increased muscle protein carbonylation and decreased Grp94 protein levels $(p<0.05)$ characterized atrophic unloaded solei. Recombinant Grp94 expression significantly reduced atrophy of transfected myofibers, compared with untransfected and empty-vector transfected ones $(p<0.01)$, and decreased the percentage of carbonylated myofibers $(p=0.001)$. Conversely, expression of two different N-terminal deleted Grp94 species did not attenuate myofiber atrophy. No change in myofiber trophism was detected in transfected ambulatory solei. The absence of effects on atrophic untransfected myofibers excluded a major role for IGFs folded by recombinant Grp94. Immunoprecipitation and confocal microscopy assays to investigate chaperone interaction with muscle atrophy regulators identified $160 \mathrm{kDa}$ neuronal nitric oxide synthase (nNOS) as a new Grp94 partner. Unloading was demonstrated to untether nNOS from myofiber subsarcolemma; here, we show that such nNOS localization, revealed by means of NADPH-diaphorase histochemistry, appeared preserved in unloaded myofibers expressing recombinant Grp94, compared to those transfected with the empty vector or deleted Grp94 cDNA $(p<0.02)$. Innovation: Grp94 interacts with nNOS and prevents its untethering from sarcolemma in unloaded myofibers. Conclusion: Maintenance of Grp94 expression is sufficient to counter unloading atrophy and oxidative stress by mechanistically stabilizing nNOS-multiprotein complex at the myofiber sarcolemma. Antioxid. Redox Signal. 20, 2479-2496.
\end{abstract}

\section{Introduction}

O XIDATIVE STRESS HAS been proposed among the factors responsible for muscle mass loss following inactivity or unloading $(31,43,47)$, two conditions that affect immobilized and/or bedridden patients. Increased levels of protein carbonylation and RNA oxidation characterize experimental animal and human unloaded muscles $(2,10,21)$, resulting in loss of biological function, which would lead to accelerated catabolism and reduced protein synthesis, respectively. In addition, the increased availability of reactive oxygen species (ROS) enhances the activity of atrophy gene regulators
$(16,37)$, and promotes the transcription of antioxidant stress-response genes and translational machinery inhibitors $(10,23,59,62)$.

Nevertheless, it remains still uncertain whether oxidative stress increases secondary to the derangement of a specific subcellular compartment or to the imbalance of myofiber antioxidant systems. Increased iron levels and byproducts of lipid peroxidation significantly accumulated in the microsomal compartment of rat soleus muscles after 12-day unloading $(21,32)$. Sarcoplasmic reticulum (SR)/sarcolemmal NADPH-oxidase and cytosolic xanthine oxidase appeared to contribute to ROS production in the diaphragm muscle

\footnotetext{
${ }^{1}$ Institute for Neuroscience, Padova Section, CNR, Padova, Italy.

${ }^{2}$ Department of Biomedical Sciences, University of Padova, Padova, Italy.
} 


\section{Innovation}

Skeletal muscle atrophy occurring after immobilization or prolonged bed rest represents a major invalidating condition. Disuse-induced disruption of neuronal nitric oxide synthase (nNOS) subsarcolemmal localization is recognized as an upstream event leading to myofiber atrophy. In this study, using an experimental animal model of muscle disuse atrophy (the hindlimb-suspended rat) and in vivo cDNA transfer, the authors identified the molecular chaperone Grp94 as a novel nNOS interacting partner, which was responsible for maintaining nNOS localization at myofiber sarcolemma and, thence, countered myofiber atrophy and oxidative stress.

exposed to prolonged mechanical ventilation $(41,72)$. Dysregulated nitric oxide (NO) production, due to untethering of neuronal nitric oxide synthase ( $\mathrm{nNOS}$ ) from sarcolemma, and increased basal hydrogen peroxide formation in inner internal membrane of mitochondria also characterized unloading and immobilization of hindlimb muscles $(42,60)$. In addition to NO (33), increased levels of cytosolic calcium trigger mitochondrial ROS production (11). Except for a contrasting report, available body of evidence suggests the occurrence of dysfunctional calcium homeostasis in disused muscles $(15,25,71,74)$.

Besides the antioxidant defense systems, a relevant role in antioxidant cytoprotection is attributed to molecular chaperones/stress proteins (27), among which the endoplasmic reticulum (ER) chaperone Grp94 distinguished itself for preventing protein carbonylation and cell death through its participation to the control of calcium homeostasis $(4,38,46$, $67)$. Grp94 binds calcium $(7,30)$ and passive ion release from the stores appears to be negatively related to the cellular content of the protein $(4,46)$.

Another interesting feature of Grp94 expression is its exclusive requirement for folding and secretion of insulin-like growth factor I and II (IGF-I and -II), the major positive autocrine regulators of muscle growth and regeneration $(63,69)$. IGF-I protein levels decreased in unloaded muscles (20), whereas increased expression of a recombinant muscle IGF-I isoform countered wasting accompanying sarcopenia and muscular dystrophies (63).

We then wondered whether Grp94 overexpression would exert an anti-atrophic role in unloaded skeletal muscle fibers, either by increasing myofiber antioxidant cytoprotection or by improving IGF maturation and release. Therefore, we investigated the effects on myofiber cross-sectional area (CSA) and presence of carbonylation after in vivo manipulation of Grp94 protein levels in soleus muscles of the tail-suspended rat. Our results indicate that increased Grp94 expression countered both oxidative stress and atrophy progression of unloaded soleus muscles, acting through an intrinsic pathway that hampered nNOS untethering from sarcolemma.

\section{Results}

\section{Grp94 expression in unloaded soleus muscles}

Soleus muscle weight/body weight ratio was used to monitor the presence of atrophy. In tail-suspended rats, it significantly decreased by about $28 \%$ after 7 -day unloading compared with ambulatory rats $\left(0.32 \times 10^{-3} \pm 0.01 \times 10^{-3}\right.$ and $0.44 \times 10^{-3} \pm 0.03 \times 10^{-3}$, mean and standard error (SE) values, respectively, $n=6, p<0.004)$, consistent with literature data $(10,26)$.

Protein carbonylation was evaluated by Oxyblot analyses (Fig. 1A). Unloaded soleus muscles displayed increased intensity on several polypeptides, compared with muscles from ambulatory rats. Densitometric analyses showed that normalized levels of protein carbonylation increased about twofold after 7 days of unloading compared with those of ambulatory muscles $(p=0.01)$.

Western blot analyses showed that the relative amount of Grp94 protein significantly decreased in unloaded soleus muscles, either using as loading control serum albumin (SA; $p=0.05$, Fig. 1B) or glyceraldehyde-3-phosphate dehydrogenase (GAPDH) (mean values \pm SE $0.44 \pm 0.05$ and $0.16 \pm 0.02$ for ambulatory and unloaded solei, respectively, $n=7$, $p=0.001$, Student's $t$-test). Consistent with previous reports $(10,23)$, protein levels of other SR/ER chaperones such as Grp78 and Grp75 did not appear to significantly change after 7 day-unloading (mean values \pm SE $0.53 \pm 0.11$ and $0.44 \pm 0.09$ for Grp78 levels of ambulatory and unloaded solei, respectively, $p=0.53$; mean values and SE $0.94 \pm 0.20$ and $0.71 \pm 0.16$ for Grp75 levels of ambulatory and unloaded solei, respectively, $p=0.42$ ), nor were affected those of calnexin and calreticulin (Supplementary Fig. S1A; Supplementary Data are available online at www.liebertpub.com/ars), whereas levels of calsequestrin (Csq) significantly increased (mean values \pm SE $0.64 \pm 0.07$ and $1.07 \pm 0.15$ for ambulatory and unloaded solei, respectively, $p=0.04$ ).

\section{In vivo transfer of Grp94 cDNA decreases myofiber atrophy}

Grp94 protein was overexpressed in soleus myofibers by means of in vivo transfection of the pT94 plasmid, which contained grp94 cDNA (67). Control transfections were performed using the empty vector codifying for green fluorescent protein ( $\mathrm{pT}$ vector). Transfected fibers were identified by the presence of the green fluorescent protein (GFP), whose cDNA was carried by the vector (Fig. 2A). The presence of recombinant Grp94 in GFP-positive fibers was visualized using a monoclonal antibody $(\mathrm{mAb})$ (clone $3 \mathrm{C} 4$; 65) that does not recognize the rat protein (67; Fig. 2B). Myofiber CSA was measured 8 days after surgery, excluding myofibers that expressed embryonic myosin ( $\varepsilon \mathrm{My})$, because of regeneration induced by the transfection protocol (Fig. 2B).

Fiber CSA of ambulatory soleus muscles was unaffected by the transfection protocol or by the presence of recombinant Grp94, because comparable values were measured in untransfected fibers and in those transfected with either $\mathrm{pT}$ or bicistronic vector codifying for recombinant Grp94 and GFP (pT94; Fig. 2C). When atrophy was induced in transfected soleus muscles by 7 days of hindlimb unloading, significantly higher CSA values were observed for recombinant Grp94expressing fibers, compared with untransfected ones and with those transfected with the empty vector $(p<0.01$; Fig. $2 \mathrm{C})$. Comparison of mean CSA values between transfected fibers of ambulatory and unloaded muscles showed that the degree of atrophy was about $28 \%$ after transfection with pT94, whereas it corresponded to $44 \%$ after transfection with the 

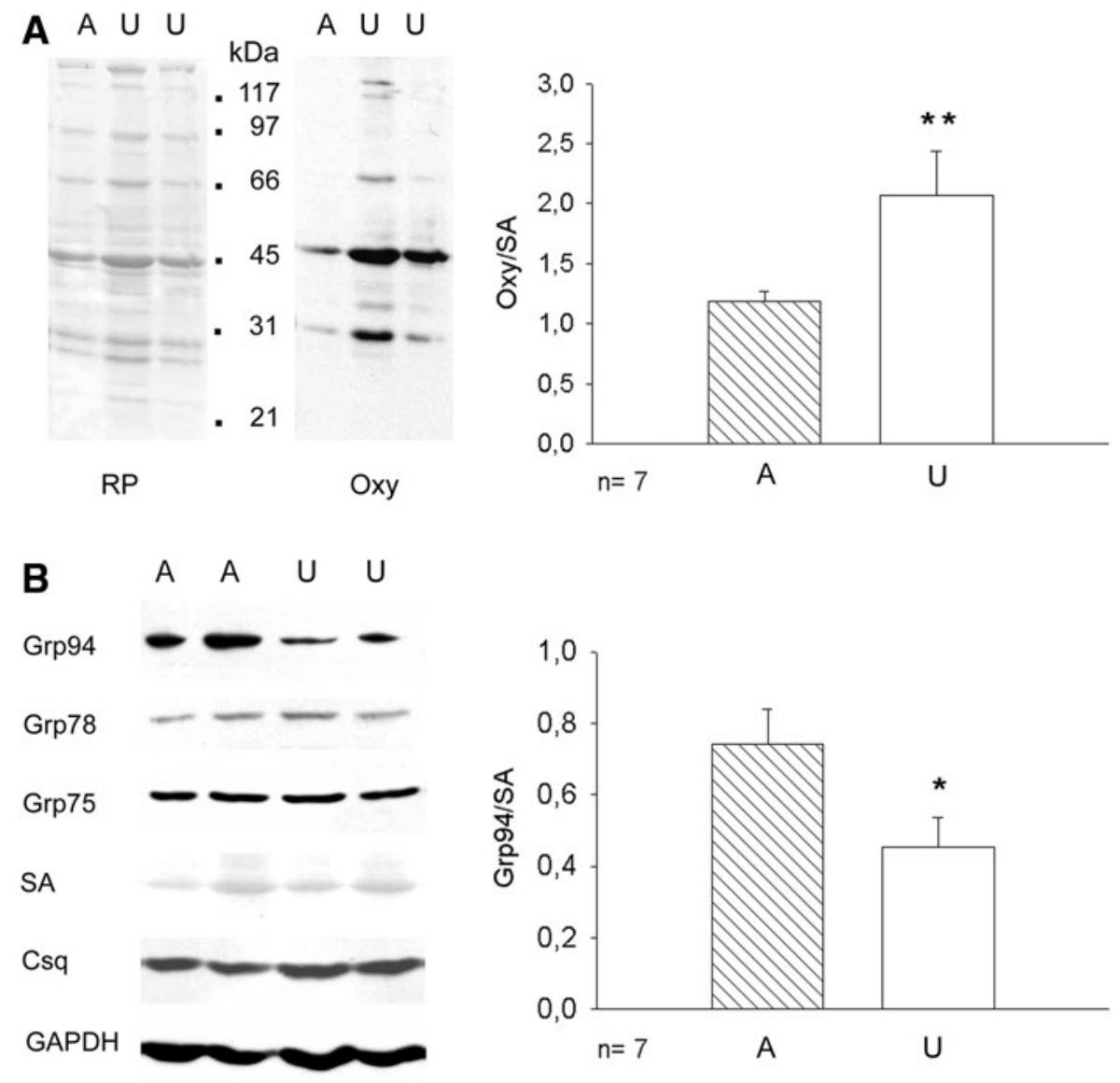

FIG. 1. Changes in protein carbonylation and Grp94 levels in unloaded soleus muscles. Dots indicate mobility of molecular weight markers (Broad Range; BioRad Lab., Rome, Italy). $n$ indicates the number of muscles evaluated in each group. ${ }^{*}$ Student's $t$-test $p=0.05 ;{ }^{* *} p=0.01$. (A) Unloading increases the degree of muscle protein carbonylation determined by Oxyblot assay, as described in "Materials and Methods". Left side: representative RP and Oxyblot (Oxy) stainings of a same blot from a 10\% acrylamide denaturing gel. Right side: bars and error bars correspond to mean and SE of densitometric values of protein carbonylation normalized to SA of ambulatory (A) and unloaded (U) muscles analyzed in parallel. (B) Unloading decreases specifically muscle levels of Grp94. Left side: Representative western blot for Grp94, Grp78, Grp75, and Csq from a 10\% acrylamide denaturing gel loaded with $50 \mu \mathrm{g}$ of whole muscle homogenates. Reference for sample loading is shown by RP staining for SA and immunolabeling for GAPDH. Right side: bars and error bars correspond to mean and SE of normalized densitometric values of samples analyzed in parallel. Csq, calsequestrin; GAPDH, glyceraldehyde-3-phosphate deydrogenase; RP, red ponceau; SA, serum albumin; SE, standard error.

empty vector. The milder degree of atrophy displayed after pT94 transfection was confirmed by distribution analysis of fiber CSA between transfected and untransfected fibers of unloaded solei (Fig. 2D). We also separately determined the effect of pT94 transfection on CSA of unloaded slow and fast myofibers, which showed a significantly higher CSA, compared with untransfected ones $(p=0.001$ for slow fibers, and $p=0.003$ for fast fibers; Fig. 2E).

Although precursor levels of the Grp94 client proteins IGF-I and II were apparently unaffected in our unloaded muscles (Supplementary Fig. S1B), their distribution was investigated by immunohistochemistry in ambulatory and unloaded transfected muscles (Supplementary Fig. S2). No difference in staining with anti-IGF-I was observed. Conversely, IGF-II immunoreactivity appeared slightly more concentrated at the subsarcolemma of ambulatory myofibers compared with unloaded ones, and again stronger in unloaded transfected myofibers expressing exogenous Grp94.
In vivo transfer of Grp94 cDNA decreases myofiber carbonylation

We investigated whether recombinant Grp94 increased myofiber antioxidant protection, using carbonyls as marker of oxidative stress. To avoid artifactual oxidation, cryosections from only two samples (usually an ambulant muscle and an unloaded one or a pT94-transfected soleus and a pT-transfected one) were collected onto the same slide, reacted with 2,4-dinitrophenylhydrazine (DNPH), and the presence of carbonyl adducts demonstrated by indirect immunoperoxidase with anti-DNPH antibodies. Soleus myofibers showed usually weak and heterogeneous DNPH immunoreactivity (Fig. 3A, upper row).

To validate visual inspection as an approach to discriminate between DNPH-positive and -negative myofibers, densitometry was performed using the Image J software on 10 positive and 10 negative fibers randomly collected from each 

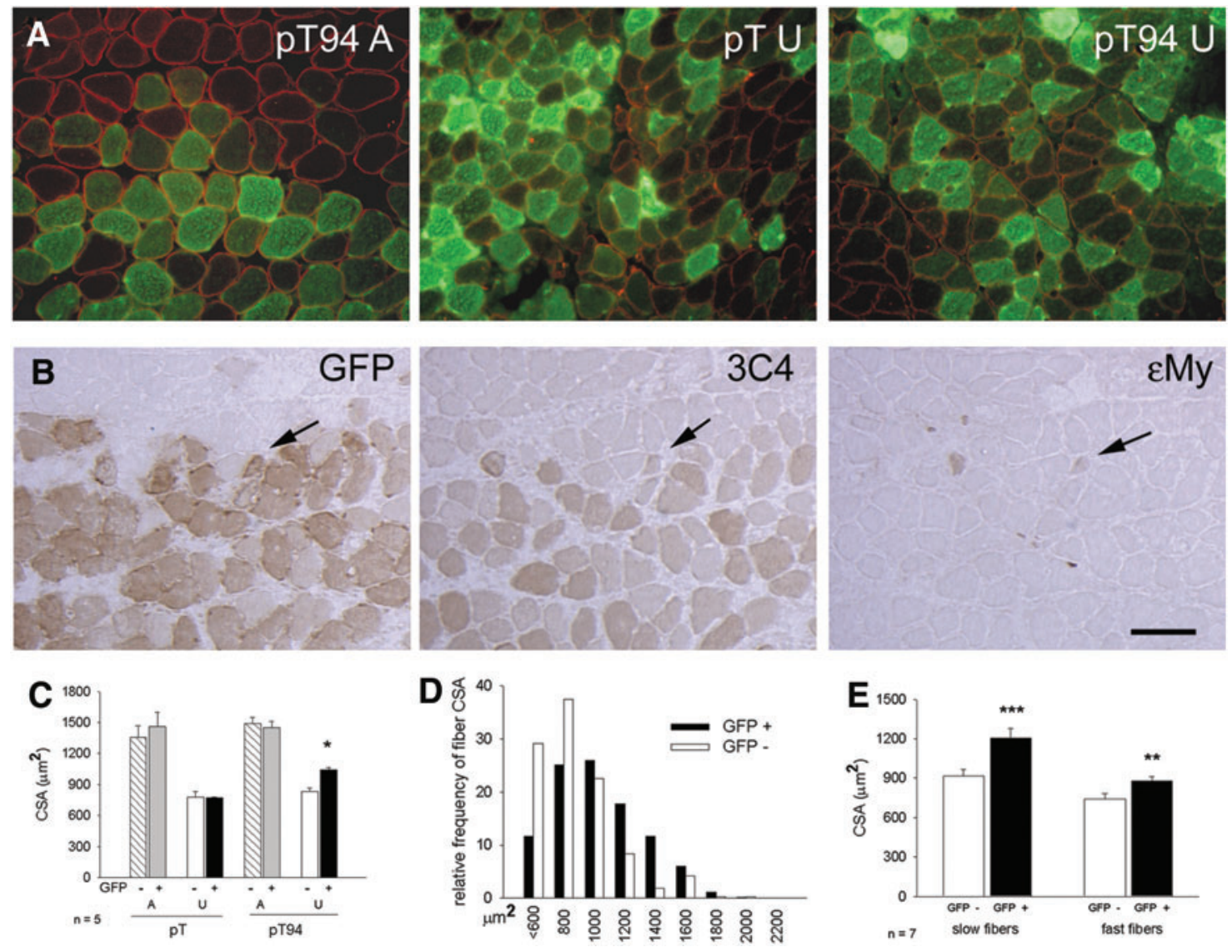

FIG. 2. Effect of recombinant Grp94 expression on myofiber CSA of unloaded soleus muscles. Bar: $100 \mu \mathrm{m}$. ${ }^{*} p<0.01$; ${ }^{* *} p=0.003$; ${ }^{* * *} p=0.001$. (A) Visualization of myofibers transfected with Grp94 cDNA (pT94) or with the empty vector (pT) by means of GFP fluorescence (sarcoplasmic fluorescence). Transverse cryosections of ambulatory (A) and unloaded (U) soleus muscles were counterstained with $\alpha$-sarcoglycan immunolabeling (sarcolemmal fluorescence) to visualize untransfected fibers for comparison of myofiber CSA. (B) Myofibers transfected with pT94 express recombinant Grp94. Serial consecutive transverse cryosections of a representative $U$ soleus muscle were labeled with indirect immunoperoxidase (dark staining) using anti-GFP antibodies, anti-Grp94 mAb 3C4, which recognizes only recombinant Grp94, and anti-eMy mAb, which identifies regenerating myofibers (indicated by arrows). Note that recombinant Grp94 is expressed in adult GFP positive myofibers. (C) Recombinant Grp94 expression attenuates CSA decrease of unloaded myofibers. Bars and error bars correspond to mean and SE values of CSA of untransfected, and pT94 and pT transfected myofibers. At least 30 myofibers (GFP+ and GFP - , each) were evaluated for a same muscle, which was considered as a single experimental unit. $n$ indicates the number of muscles evaluated in each group. Unloading significantly decreased CSA compared to A myofibers. Asterisk indicates the presence of significant difference versus CSA values of untransfected fibers of the same pT94 transfected muscles and versus those of $\mathrm{pT}$-transfected and untransfected fibers of muscles obtained from U rats matched on body weight (Withinsubject ANOVA and Bonferroni post-hoc analysis). (D) Myofiber CSA distribution from seven pT94 U solei. Relative frequency histogram of CSA values of Grp94-transfected fibers $(n=662)$ and of untransfected ones $(n=480)$. (E) Recombinant Grp94 expression attenuates unloading-induced CSA decrease of both slow and fast myofibers. Bars and error bars correspond to mean and SE values of CSA of pT94 transfected and untransfected myofibers of unloaded muscles. At least 50 myofibers (GFP + and GFP - , each) were evaluated for the slow fiber type in a same muscle, which was considered as a single experimental unit. (Paired Student's $t$-test). At least 20 myofibers (GFP + and GFP - , each) were evaluated for the fast fiber type (Paired Student's $t$-test). ANOVA, analysis of variance; CSA, cross-sectional area; GFP, green fluorescent protein; $\mathrm{mAb}$, monoclonal antibody; My, myosin heavy chain; $\mathrm{pT}$, empty vector codifying for GFP; pT94, bicistronic vector codifying for recombinant Grp94 and GFP. To see this illustration in color, the reader is referred to the web version of this article at www.liebertpub.com/ars

soleus muscle of five ambulatory and five unloaded rats. Mean optical density (OD) of DNPH-positive fibers was significantly higher than that of negative ones in both unloaded and ambulatory muscles (mean and SE values of absorbance of DNPH-positive and -negative fibers of unloaded muscles were $118.20 \times 10^{-4} \pm 8.78 \times 10^{-4}$ and $106.15 \times 10^{-4} \pm 5.07 \times 10^{-4}$, respectively, $p=0.013$; mean and SE values of absorbance of DNPH-positive and -negative fibers of ambulatory muscles were $113.43 \times 10^{-4} \pm 5.74 \times 10^{-4}$ and $102.02 \times 10^{-4} \pm 6.00 \times 10^{-4}$ respectively, $p=0.0019$, paired Student's $t$-test).

About $20 \%$ of total myofibers of ambulatory soleus muscles displayed positive immunoreactivity for DNPH. These reactive fibers corresponded to about $50 \%$ of the fast fiber population (Fig. 3A, large arrowheads, B) and less than $10 \%$ of the slow fiber one. In unloaded muscles, staining for DNPH involved about $40 \%$ of total myofibers, corresponding to about 
A

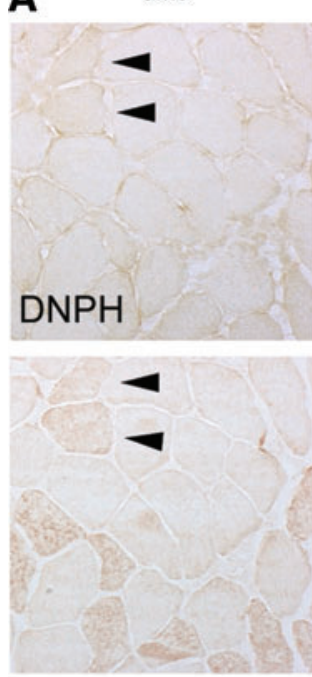

U

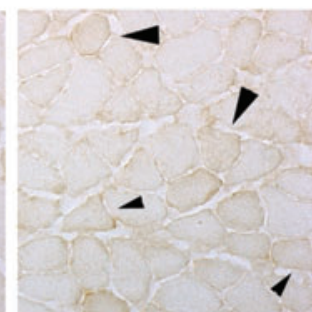

рT94 U

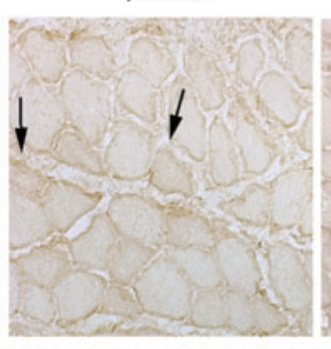

PT U
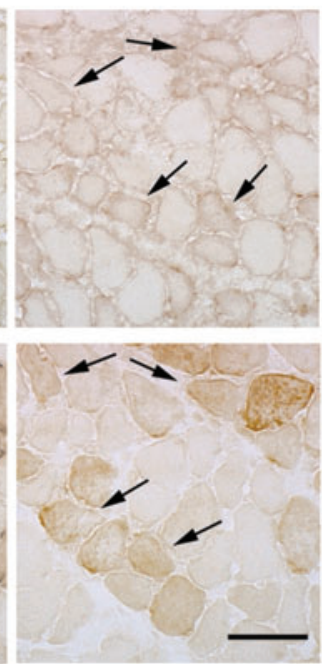

GFP
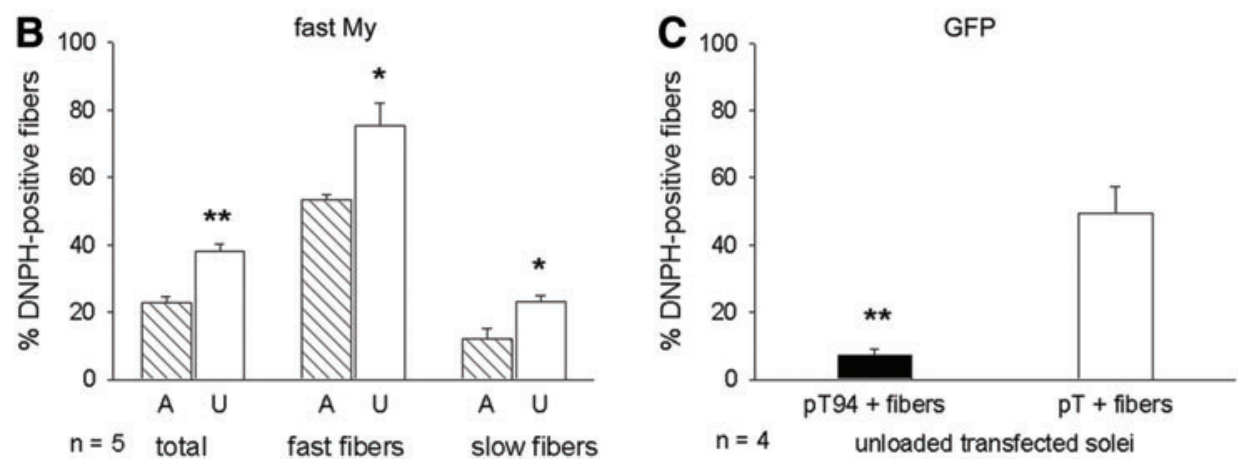

FIG. 3. Effect of recombinant Grp94 expression on myofiber carbonylation of unloaded soleus muscles. *Student's $t$-test $p<0.02$; ${ }^{* *} p<0.001$. (A) Presence and distribution of myofiber carbonylation vary after unloading and pT94 transfection. Consecutive transverse cryosections of ambulatory (amb) or unloaded (U) soleus muscles were exposed to DNPH, as described in "Materials and Methods", and labeled with anti-DNPH antibodies (upper row) or stained for fast My (lower row) in indirect immunoperoxidase. Large and small arrowheads indicate representative DNPH-positive myofibers of the fast and slow fiber populations, respectively. Similarly, cryosections from U solei transfected with either Grp94 cDNA (pT94) or empty vector (pT), were reacted for DNPH (upper row) or labeled with anti-GFP antibodies (lower row). Arrows indicate DNPH-positive transfected myofibers. Bar: $50 \mu \mathrm{m}$. (B) Hindlimb unloading increases the percentage of carbonylated myofibers. Bars and error bars correspond to mean and SE values of the percentage of DNPH positive fibers evaluated on the total myofiber amount of ambulatory (A) and $U$ solei, and, separatedly, on their respective fast and slow fiber populations. Average $n$ of fibers considered for each muscle: 350. $n$ indicates the number of muscles evaluated in each group. (C) Transfection with pT94 decreases the percentage of DNPH-positive myofibers in unloaded solei. Bars and error bars correspond to mean and SE values of the percentage of DNPH-positive fibers observed among myofibers transfected with pT94 or with pT. Average $n$ of transfected fibers evaluated in each muscle: 80 . DNPH, 2,4-dinitrophenylhydrazine. To see this illustration in color, the reader is referred to the web version of this article at www.liebertpub.com/ars

$75 \%$ of the fast fiber population and significantly doubling their percentage within the slow fiber population (Fig. 3A, small arrowheads, $\mathrm{B} ; p<0.02)$. In unloaded transfected muscles, the distribution of DNPH immunoreactivity was analyzed considering GFP-positive and GFP-negative fibers (Fig. 3A). The percentage of unloaded transfected fibers showing DNPH immunoreactivity was sevenfold less after transfection with pT94, the large majority appearing unstained, at variance with myofibers transfected with the empty vector (Fig. 3C, $p=0.001$ ).

Similarly, a lower percentage of DNPH-positive myofibers was detected among pT94 transfected myofibers of ambulatory muscles, compared to untransfected ones (Supplementary Fig. S3).

\section{No effect on myofiber atrophy after expression of N-terminal deleted Grp94}

To validate the involvement of Grp94 in attenuation of muscle mass loss, transfection was performed using constructs (pVSV-G5B and vector codifying for VSV-G tagged N-deleted Grp94 [pVSV-G6B]) that codified for deleted N-terminal Grp94 forms. The former expressed a $\Delta 1-271$ Grp94 protein that lacked the nucleotide binding domain (Supplementary Fig. S4A); the latter expressed a $\Delta 1-570$ Grp94 form, which lacked three $\mathrm{N}$-terminal putative protein-binding sites $(3,58)$, but retained one (AA 652-754) (73). The $\Delta 1-570$ Grp94 protein also participates to calcium homeostasis in a manner similar to the full-length Grp94 (46 and P. Pizzo, 
C. Scapin and L. Gorza, unpublished observations). The expression of the exogenous proteins was monitored by the presence of the VSV-G tag, which was added to the construct at the N-terminal region, immediately downstream the signal peptide required for the ER import. Figure 4A shows representative VSV-G immunohistochemistry of transfected ambulatory soleus muscles and 7-day unloaded ones. Fiber CSA of ambulatory soleus muscles was unaffected by the expression of the deleted Grp94 forms, either considering fast or slow fiber populations (Fig. 4B). A comparable result was observed for unloaded myofibers, whose CSA appeared reduced compared to myofiber values of ambulatory muscles, independently from the presence of deleted Grp94 $(p<0.01$; Fig. 4B).

\section{nNOS interacts with Grp94}

The effect of recombinant Grp94 expression upon atrophy and carbonylation appeared circumscribed to transfected fibers, therefore excluding a major mechanistical contribution through increased IGF secretion (53) and suggesting the chaperone involvement in other pathways. Immunoprecipitation experiments, performed using two different anti-Grp94 antibodies to explore physiological interactions with molecules promoting myofiber atrophy development, showed that Grp94 co-immunoprecipitated from lysates of both skeletal muscle and cerebellum with a $160 \mathrm{kDa}$ polypeptide recognized by anti nNOS antibodies (Fig. 5A). Similarly, anti-nNOS polyclonal antibodies (pAbs) immunoprecipitated Grp94, but not Grp75,

\section{A pVSV-G 5B (41-271 Grp94)}
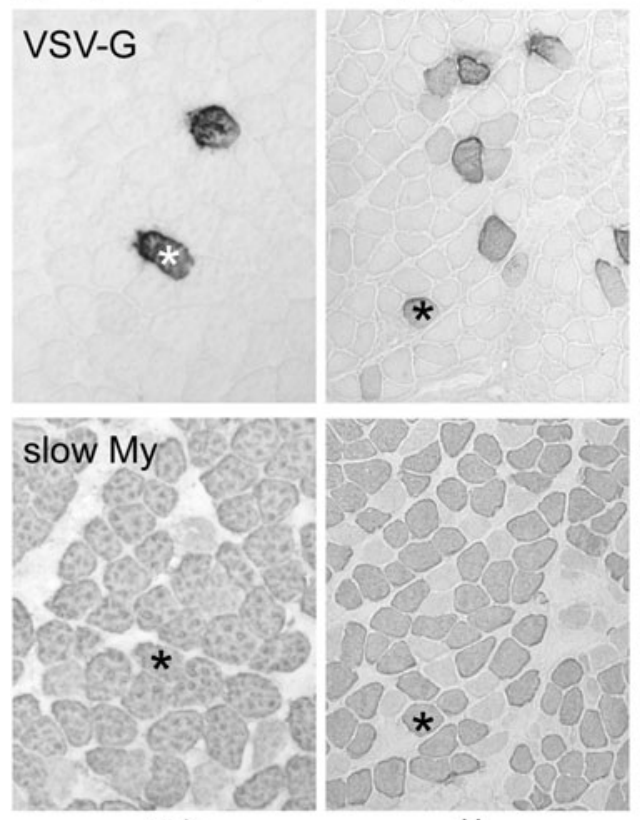

$\mathrm{amb}$
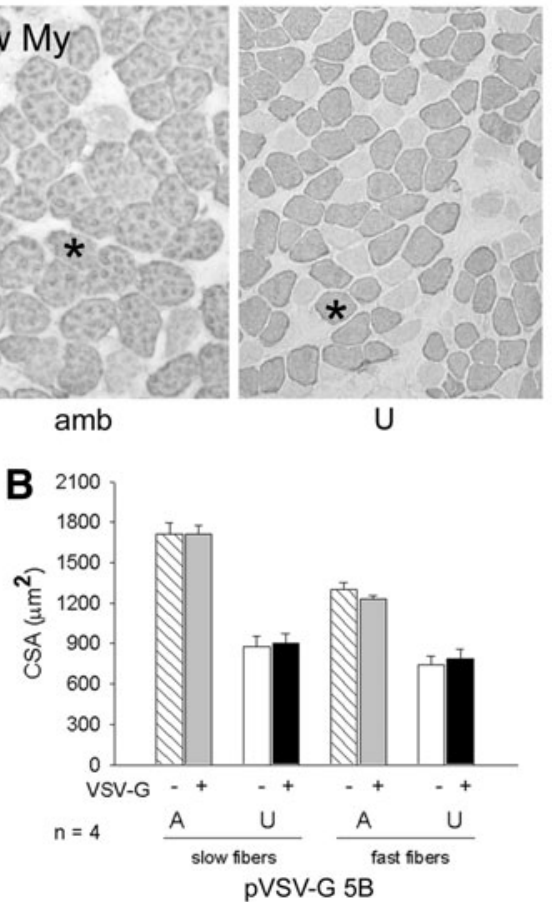

pVSV-G 6B ( $(11-570$ Grp94)
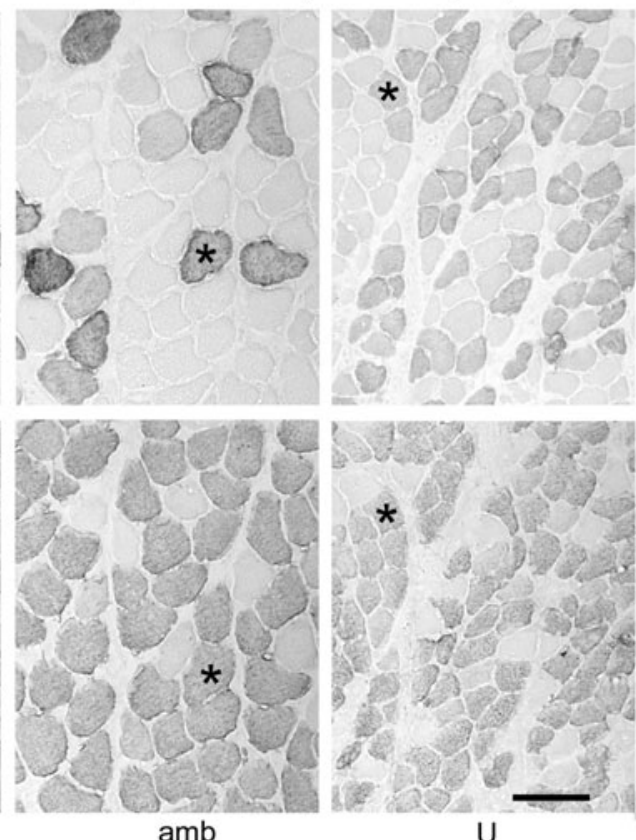

$\mathrm{amb}$

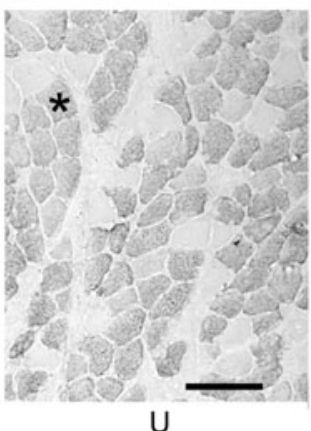

U

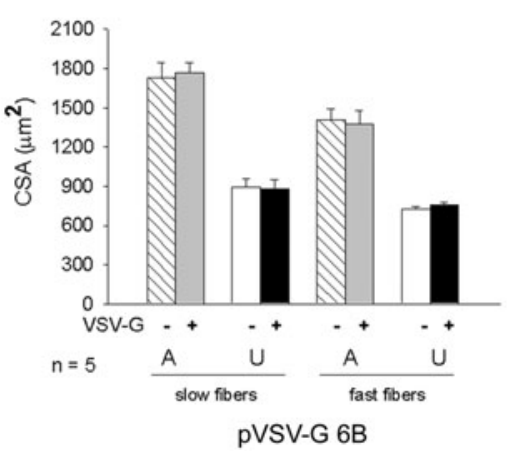

FIG. 4. Effect of N-ternimal deleted Grp94 expression on myofiber CSA of unloaded soleus muscles. (A) Visualization of the expression of two different N-deleted Grp94 proteins in transfected ambulatory (amb) and unloaded (U) soleus muscles. Indirect immunoperoxidase on consecutive transverse cryosections labeled with anti-VSV-G antibody, which recognizes a tag in the recombinant deleted Grp94 forms or with anti-slow My antibody. Asterisks identify transfected slow myofibers. Bar: $100 \mu \mathrm{m}$. (B) CSA values of VSV-G-negative and -positive myofibers in the slow and fast fiber populations of ambulatory (A) and U muscles. Bars and error bars correspond to mean and SE values. At least 30 myofibers (VSV-G+ and VSV-G-, each) were evaluated for fiber type in a same muscle, which was considered as a single experimental unit. $n$ indicates the number of muscles evaluated in each group. Unloading significantly decreased CSA of both transfected and untransfected fibers compared to A ones of muscles obtained from rats matched on body weight (within-subject ANOVA and Bonferroni post-hoc analysis $p<0.01$ ). 


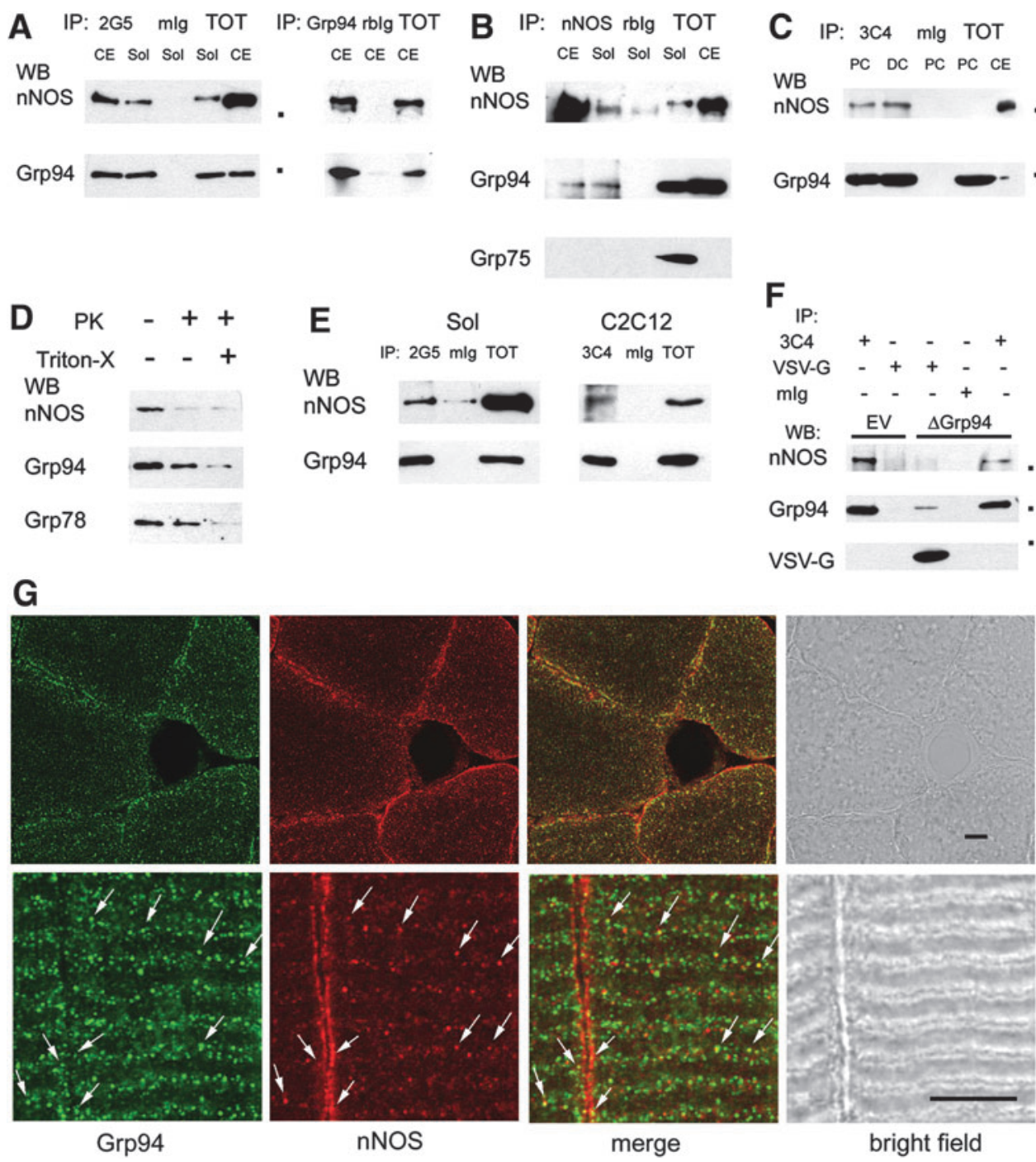

FIG. 5. Co-IP and co-localization of Grp94 with nNOS. Dots indicate mobility of molecular weight markers (from the top: 150,000; 100,000; 37,000 Da, Precision Plus; BioRad). Experimental preparations and IPs were repeated at least three times. (A) Grp94 immunoprecipitates with nNOS. Representative western blots of $7.5 \%$ acrylamide gels loaded with CE and Sol protein lysates, before (TOT) and after immunoprecipitation (IP) with two different anti-Grp94 antibodies (2G5 mAb on the left side and anti-Grp94 pAb on the right side); $\mathrm{mIg}$ and rbIg indicate IP with non-immune immunoglobulins. Anti-nNOS mAb and anti-Grp94 $\mathrm{pAb}$ were used for nitrocellulose staining. (B) nNOS immunoprecipitates with Grp94. Representative western blot of a 7.5\% acrylamide gel loaded with CE and Sol protein lysates, before and after IP with nNOS pAb. Nitrocellulose staining with anti-Grp75 $\mathrm{mAb}$ is shown in addition to $\mathrm{nNOS}$ and Grp94 labeling. (C) Grp94 and nNOS co-immunoprecipitate from cross-linked C2C12 cells. Representative western blots of 7.5\% acrylamide gels loaded with protein lysates obtained after chemical cross-linking of proliferating (PC) and $6 \mathrm{~h}$ differentiating (DC) $\mathrm{C} 2 \mathrm{C} 12$ cultures, before and after IP with the anti-Grp94 mAb 3C4. Anti-nNOS mAb and anti-Grp94 pAb were used for nitrocellulose staining. (D) Grp94 and nNOS topology in rat soleus microsomes. Representative western blot of a $7.5 \%$ acrylamide gel loaded with $50 \mu \mathrm{g}$ of crude membranes fractionated from ambulatory soleus muscles, as described in "Materials and Methods," and exposed or not to $200 \mathrm{ng} / \mathrm{ml}$ of PK, in the absence or in the presence of $0.5 \%$ Triton-X for $30 \mathrm{~min}$ at RT. Nitrocellulose strips were stained for nNOS, Grp94, and Grp78. (E) Grp94 co-immunoprecipitates with nNOS from cross-linked microsomes. Representative western blots of $7.5 \%$ acrylamide gels loaded with crude membranes fractionated from ambulatory soleus muscles and cross-linked in vitro before lysis, as described in "Materials and Methods," (left side), or from crosslinked C2C12 cultures (right side). Microsomes were loaded before and after IP with anti-Grp94 mAbs 2 G5 (left side) or 3C4 (right side). Anti-nNOS mAb and anti-Grp94 pAb were used for nitrocellulose staining. (F) $\Delta 1-570$ Grp94 does not immunoprecipitate nNOS. C2C12 cells transiently transfected with pVSV-G6B ( $\Delta$ Grp94), or with the empty vector (EV), were cross-linked before lysis and immunoprecipitated with anti-Grp94 mAb 3C4 and anti-VSV-G mAb. Nitrocellulose strips from a representative western blot of $6-12 \%$ acrylamide gradient gel were labeled with anti-nNOS mAb, anti-Grp94 pAb, and anti-VSV-G mAb. (G) Grp94 and nNOS co-localization. Confocal microscopy images of transverse (upper row) and longitudinal sections (lower row) of soleus muscle after double immunofluorescence staining with anti-nNOS pAb (red fluorescence) and anti-Grp94 mAb 2G5 (green fluorescence). Arrows point to sites of colocalization of $\mathrm{nNOS}$ and Grp94 (yellow fluorescence), which appear on globular structures mainly present at the A-I boundary and at the sarcolemma. Bars: $5 \mu \mathrm{m}$. CE, cerebellum; DC, differentiating C2C12 cells; mIg, mouse immunoglobulin; nNOS, neuronal nitric oxide synthase; PC, proliferating C2C12 cells; rbIg, rabbit immunoglobulin; Sol, soleus muscle; TOT, total lysate. 
another ER chaperone/stress protein involved in antioxidant cytoprotection and known to interact with Grp94 (10, 29; Fig. 5B). Specificity of the antibodies used is illustrated in Supplementary Figure S4B.

In order to exclude the aspecific nature of such interaction, cultures of the murine myogenic cell line $\mathrm{C} 2 \mathrm{C} 12$ were exposed to a cell-permeant protein cross-linker before lysis and Grp94 was immunoprecipitated using a third different anti-Grp94 $\mathrm{mAb}(19,66)$. Western blot analysis confirmed the presence of interaction of the chaperone with $\mathrm{nNOS}$, in both proliferating and differentiating C2C12 myoblasts (Fig. 5C).

Since these results suggested an interaction between a cytosolic protein (nNOS) and an essentially lumenal one (Grp94), topology of this interaction was explored using crude membrane preparations obtained from soleus muscle of ambulatory rats (Fig. 5D). Microsomes were exposed to proteinase $\mathrm{K}(\mathrm{PK})$ digestion in the absence or presence of Triton- $X$ (14). Addition of $200 \mathrm{ng} / \mathrm{ml} \mathrm{PK}$ was sufficient to abolish nNOS immunoreactivity, whereas the presence of $0.5 \%$ Triton-X was required to blunt Grp94 immunostaining, which however appeared already reduced after exposure to PK alone. Immunostaining of the same blots for another lumenal protein, such as Grp78, revealed a $25.00 \pm 5.85 \%$ less decrease in labeling intensity (mean and SE of three different experi- ments), suggesting that a proportion of Grp94 molecules assumed a transmembrane localization (see also Supplementary Fig. S4C). Cross-linked microsomes, either directly prepared on crude membrane fraction obtained from soleus muscle or fractionated from cross-linked $\mathrm{C} 2 \mathrm{C} 12$ cultures, demonstrated further co-immunoprecipitation of nNOS and Grp94 (Fig. 5E).

To ascertain whether the interaction with nNOS occurred also with $\Delta 1-570$ Grp94 form, immunoprecipitation assays were performed on lysates obtained from C2C12 cells after transient transfection with pVSV-G $6 \mathrm{~B}$ and chemical crosslinking. $\Delta 1-570$ Grp94, immunoprecipitated by anti-VSV-G antibodies, showed an apparent $\mathrm{Mr}$ of 34,000 Da and did not co-immunoprecipitate nNOS, at variance with immunoprecipitated endogenous Grp94 from parallel aliquots of the same lysates and from $\mathrm{C} 2 \mathrm{C} 12$ cells transfected with the empty vector (Fig. 5F).

Analysis of the subcellular distribution of nNOS and Grp94 was performed by confocal microscopy on both transverse and longitudinal cryosections of soleus muscles (Fig. 5G). Grp94 labeling, obtained using two different anti-Grp94 antibodies, concentrated at the subsarcolemmal region and extended amid myofibrils (Fig. 5G, upper panel, green fluorescence, and not shown), diffusely staining the I band, but

FIG. 6. Effects of the expression of recombinant or deleted Grp94 on subsarcolemmal nNOS localization of unloaded soleus muscles. Bar: $25 \mu \mathrm{m}$. ${ }^{*} p<0.01 ;{ }^{* *} p<0.001$. (A) Sarcolemmal nNOS immunolabeling in rat soleus muscles. Transverse cryosections of ambulatory (A) and unloaded (U) soleus muscle were reacted with anti-nNOS pAb (red fluorescence) and nuclei counterstained with DAPI (blue fluorescence). Arrows point to reactive, discrete subsarcolemmal regions, which appear reduced by unloading. (B) Western blot for $\mathrm{nNOS}$ in rat soleus muscles. Representative blot from a $8 \%$ acrylamide denaturing gel loaded with $100 \mu \mathrm{g}$ of whole muscle homogenates. Dots indicate mobility of molecular weight markers (from the top: 250,000 and 150,000). Reference for sample loading is shown by RP staining for SA. (C) Sarcolemmal nNOS activity, revealed by NADPH-d histochemistry as detailed in "Materials and Methods," remains detectable in unloaded myofibers after transfection with grp94 cDNA (pT94). Consecutive transverse cryosections were used for NADPH-d enzyme histochemistry (dark blue, upper row) and immunoperoxidase staining with anti-GFP antibodies, to identify transfected myofibers (brown staining, lower row). pT indicates the empty vector. Asterisks indicate representative transfected fibers. Note the presence of discontinuous NADPH-d reactivity at the myofiber sarcolemma of A solei. (D) Recombinant Grp94 expression prevents the unloading-induced loss in sarcolemmal NADPH-d reactivity. Bars and error bars correspond to mean and SE percentage of fiber CSC positive for NADPH-d enzyme histochemistry. Asterisks indicate the presence of significant statistical difference versus both transfected and untransfected myofibers of pT94A muscles and versus GFP+ myofibers of pT94U muscles obtained from rats matched on body weight $(p<0.01$; within-subject ANOVA test Bonferroni post-hoc test); average $n$ of transfected or untransfected fibers with detectable sarcolemmal NAPDH-d reactivity evaluated in a same muscle: 15 each. $n$ indicates the number of muscles evaluated in each group. (E) Distribution of CSC percentage corresponding to positive sarcolemma for NADPH-d enzyme histochemistry among pT94 transfected fibers $(n=79)$ and untransfected ones $(n=66)$ obtained from $7 \mathrm{U}$ muscles. (F) Recombinant Grp94 expression prevents the unloading-induced increase in intensity of sarcoplasmic NADPH-d enzyme histochemistry. Bars and error bars correspond to mean and SE values of OD. Asterisks indicate the presence of significant statistical difference between untransfected myofibers of A and U muscles (Student's $t$ test) and between untransfected and pT94-transfected myofibers of U muscles (paired Student's $t$-test); average $n$ of fibers evaluated for each group: 30 from 3 different muscles. (G) $\Delta 1-570$ Grp94 expression does not prevent the loss in sarcolemmal NADPH-d enzyme histochemistry. Bars and error bars correspond to mean and SEM percentage of fiber CSC positive for NADPH-d staining. About 15 transfected (VSV-G+) and untransfected (VSV-G-) fibers with detectable sarcolemmal NAPDH-d activity were evaluated in each muscle. Unloading significantly decreased CSC of both transfected and untransfected fibers compared to A ones evaluated from muscles obtained from rats matched on body weight (Within-subject ANOVA and Bonferroni post-hoc analysis $p<0.01)$. (H) Triple immunofluorescence staining of representative transverse cryosections of pT94-transfected unloaded soleus muscles with anti-FOXO3a antibodies (red fluorescence), anti- $\alpha$-sarcoglycan antibody (sarcolemmal green fluorescence). and DAPI (blue fluorescence). Sarcoplasmic green fluorescence corresponds to GFP and identifies transfected myofibers. Arrows indicate a representative FOXO3a positive nucleus in an untransfected myofiber (pink fluorescence after merging with DAPI). Arrowheads point to nuclei in transfected myofibers that are unstained for FOXO3a. Histograms represent quantitative evaluation of FOXO3a-positive nuclei in myofibers of unloaded solei in the presence and in the absence of pT94 transfection. Bars and error bars correspond to mean and SE values of the percentage of FOXO3a-positive nuclei evaluated on the total amount of myonuclei detected in transfected and in untrasfected myofibers. Data were collected from six micrographic fields from two muscles for a total number of 400 myofiber nuclei. CSC, crosssectional circumference; DAPI, 4',6-diamidino-2-phenylindole; NADPH-d, NAPDH diaphorase; OD, optical density. 
the $\mathrm{Z}$ line (Fig. 5G, lower panel, and not shown). Anti-nNOS antibodies strongly labeled the subsarcolemmal region and weakly the sarcomeric I band (Fig. 5G, red fluorescence). Colocalization of Grp94 and nNOS signals involved a minor population of globular structures, adjacently to the A-I boundary and sarcolemma (Fig. 5G, arrows).

\section{Grp94 upregulation recovers subsarcolemmal NADPH-diaphorase activity in unloaded muscles}

nNOS untethering from sarcolemma represents a major initiating event leading to disuse muscle atrophy (60). Antibodies to nNOS stained sarcolemmal portions in cross sections of ambulant rat muscles (Figs. 5G, upper row, and 6A, arrows; 50), whereas immunolabeling at these sites appeared grossly reduced in unloaded solei (Fig. 6A), although western blot analyses (Fig. 6B) did not reveal any change in the relative amount of the protein (mean values \pm SE $0.77 \pm 0.13$ and $0.73 \pm 0.13$ for ambulatory and unloaded solei, respectively, $n=5, p=0.83$, Student's $t$-test).

A consistent distribution was observed using the histochemical reaction for NAPDH-diaphorase (NADPH-d) (50), which had the advantage to reveal the presence of the active enzyme compared to immunolocalization (Fig. 6C). In ambulatory soleus muscle, staining appeared concentrated in discrete sarcolemmal regions corresponding to about $50-60 \%$ of fiber cross-sectional circumference (CSC). Enzyme histochemistry for NADPH-d was then used to evaluate the percentage of sarcolemma that bound active nNOS in transfected myofibers, identified in serial consecutive
A
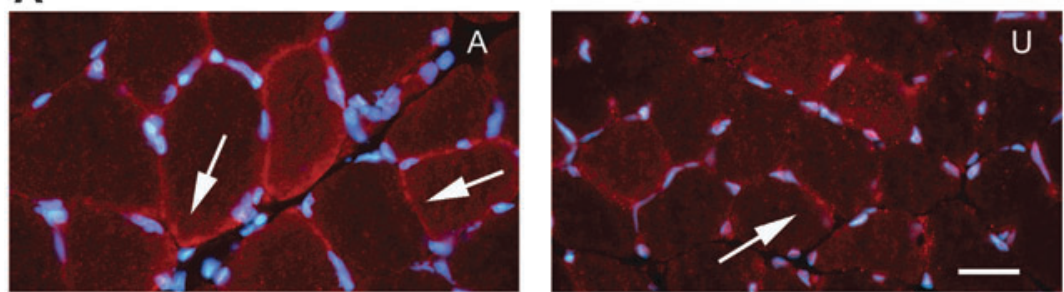

u

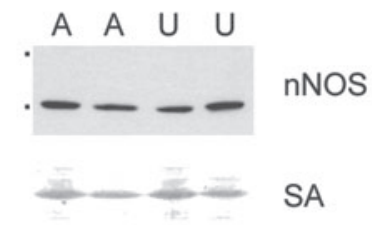

C

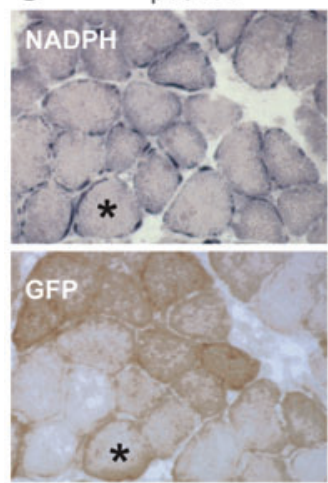

RTugru

pT94 U

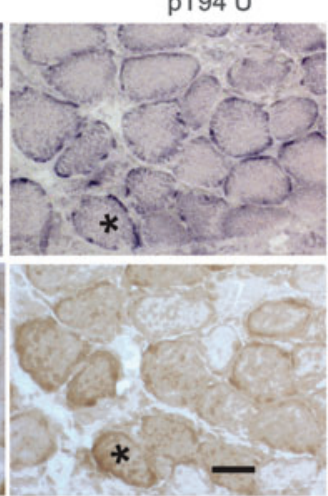

D

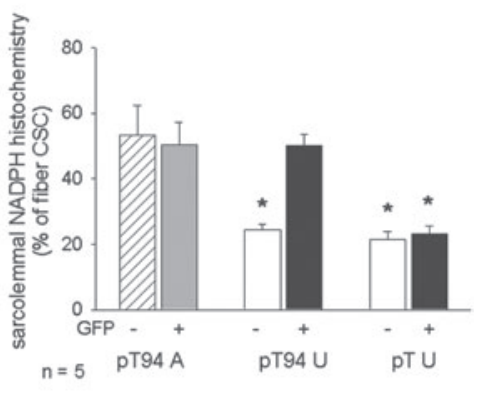

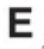

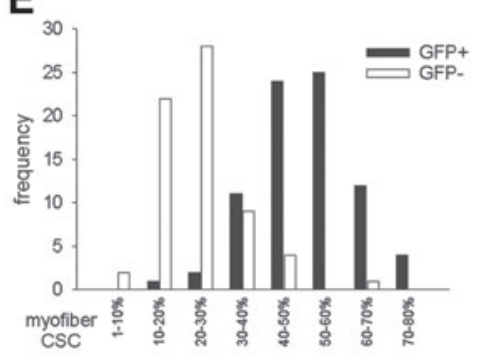

$\mathbf{F}$
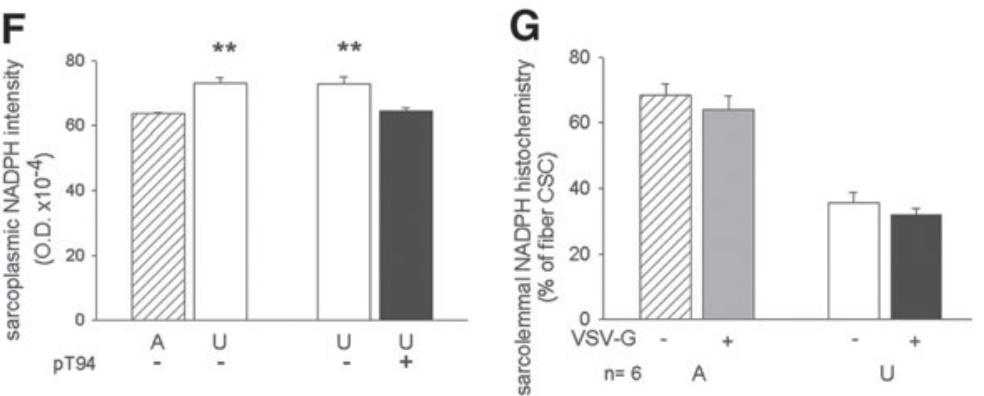

H

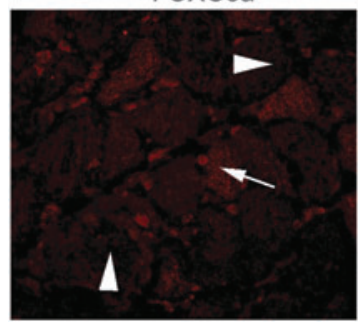

FOXO3a/ $\alpha$ sarc-GFP(pT94)

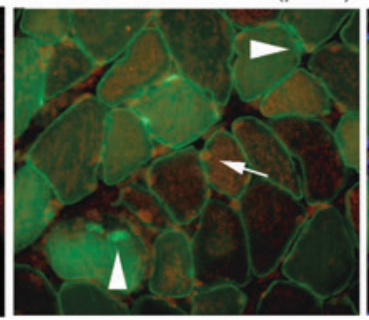

merged with DAPI

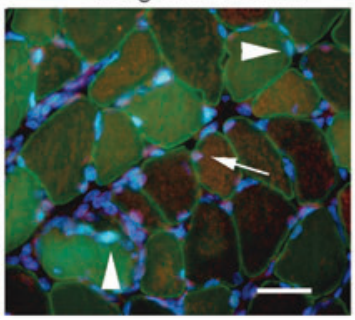

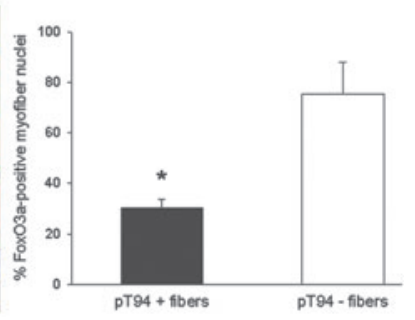


sections using anti-GFP antibodies and indirect immunoperoxidase. Sarcolemmal distribution of NADPH-d activity appeared significantly reduced in unloaded myofibers, either transfected with the empty vector or untransfected ones, compared to ambulatory muscles $(p<0.01)$, whereas it appeared unaffected in myofibers transfected with pT94 (Fig. 6C-E).

On the other hand, the decreased sarcolemmal distribution observed in unloaded myofibers was accompanied by a significant, albeit slight, increased intensity of sarcoplasmic NADPH-d enzyme histochemistry, which was indicative of accumulated active nNOS (Fig. 6F). Transfection with exogenous Grp94 significantly decreased intensity of sarcoplasmic NADPH-d activity in unloaded muscles (Fig. 6F).

The same analysis on sarcolemmal distribution of NADPH-d activity was repeated in muscles transfected with the construct codifying for $\Delta 1-570$ Grp94. Unloaded untransfected myofibers showed a significantly reduced percentage of CSC positive for sarcolemmal NADPH-d activity, which, however, did not increase after transfection with pVSV-G 6B $(p<0.01$, Fig. 6G).

Since our transfection protocol allowed analyses at the fiber level only, no biochemical assay could be performed to investigate the effect of Grp94 overexpression on nNOSreleased NO endpoints in unloaded muscles. Nevertheless, immunofluorescence studies revealed a negative effect on pro-catabolic signals, such as reduced nuclear localization of FoxO3a and ubiquitin labeling, in pT94-transfected myofibers (Fig. 6H; Supplementary Fig. S5).

\section{nNOS mediates the trophic effects of Grp94 upregulation in unloaded muscles}

To demonstrate that Grp94 acted upstream nNOS dysregulation in unloaded muscles, the specific nNOS inhibitor 7-nitroindazole (7-NI) was administered to ambulatory and hindlimb-suspended rats (60). Control rats received peanut oil only. Seven-day treatment almost abolished subsarcolemmal NADPH-d enzyme histochemistry in ambulatory muscles (Fig. 7A), without affecting both subsarcolemmal $\mathrm{nNOS}$ localization (not shown) and the co-immunoprecipitation with Grp94 (Fig. 7B).

Consistent with Suzuki et al. (60), 7-NI treatment during hindlimb unloading significantly attenuated myofiber atrophy of soleus muscle, compared with oil-treated unloaded rats, (Fig. 7A, C; analysis of variance [ANOVA] $p=0.003$ ). Further, it affected myofiber carbonylation. The percentage of DNPH-positive myofibers remained at ambulatory level in 7-NI-treated unloaded solei, at variance with oil-treated unloaded muscles, where they significantly increased $(p<0.01$; Fig. 7D, E). Similarly, no significant increase in protein carbonylation levels was observed by Oxyblot analysis between ambulatory and unloaded solei of 7-NI-treated rats (Supplementary Fig. S6).

Eventually, transfection with pT94 of soleus muscles of unloaded rats treated with 7-NI did not significantly increase further CSA of either slow or fast myofibers, compared to oiltreated unloaded rats, proving that $\mathrm{nNOS}$ is the mediator of the trophic effects of exogenous Grp94 expression in unloaded muscles (Fig. 7F, G). Expression of recombinant

FIG. 7. Effects of the expression of recombinant Grp94 in unloaded solei after inhibition of nNOS with 7-NI. Dots indicate mobility of molecular weight markers (from the top: 150,000; 100,000 Da). Bar: $50 \mu$ m. ${ }^{*} p<0.05 ;{ }^{* *} p<0.01,{ }^{* * * *} p<0.005$. (A) Sarcolemmal nNOS activity, revealed by NADPH-d histochemistry, disappeared after treatment with 7-NI. Transverse cryosections of ambulatory (A) and unloaded (U) soleus muscles obtained from rats treated with vehicle (OIL) or with 7-NI were used for NADPH-d histochemistry. Note the presence of positive, discontinuous, NADPH-d activity at the myofiber sarcolemma of A OIL solei. (B) Grp94 immunoprecipitates nNOS in 7-NI solei. Representative western blots of 7.5\% acrylamide gels loaded with immunoprecipitates (IP) of protein lysates from ambulatory solei of OIL and 7-NI treated rats, obtained using anti-Grp94 mAb 2G5 or non-immune mIg. Anti-nNOS mAb and anti-Grp94 pAb were used for nitrocellulose staining. (C) 7-NI treatment attenuates CSA decrease of unloaded myofibers. Bars and error bars correspond to mean and SE values of myofiber CSA of A and U solei from OIL and 7-NI treated rats. At least 30 myofibers were evaluated for each muscle, which was considered as a single experimental unit. $n$ indicates the number of muscles evaluated in each group. Asterisks indicate significant difference between U groups and versus the respective A muscle (ANOVA and Bonferroni posthoc analysis). (D) Presence of myofiber carbonylation in U solei of 7-NI treated rats. Transverse cryosections were exposed to DNPH, as described in "Materials and Methods," and labeled with anti-DNPH antibodies using indirect immunoperoxidase. Arrows indicate representative carbonylated myofibers. (E) 7-NI treatment decreases the percentage of carbonylated myofibers in unloaded muscles. Bars and error bars correspond to mean and SE values of the percentage of DNPH-positive fibers evaluated on the total myofiber amount of A and U solei of OIL and 7-NI treated rats, and, separatedly, on their respective fast and slow fiber populations. Average $n$ of fibers considered for each muscle: 700. (ANOVA and Bonferroni post-hoc analysis). (F) Visualization of myofibers transfected with Grp94 cDNA (pT94) by means of GFP fluorescence (sarcoplasmic fluorescence) in unloaded muscles of OIL and 7-NI treated rats. Cryosections were counterstained with $\alpha$-sarcoglycan immunolabeling (sarcolemmal fluorescence) to visualize untransfected fibers for comparison of myofiber CSA. (G) Recombinant Grp94 expression does not affect CSA of unloaded myofibers of 7-NI-treated rats. Bars and error bars correspond to mean and SE values of CSA of pT94 transfected and untransfected slow and fast myofibers of unloaded muscles of OIL and 7-NI-treated rats. At least 20 myofibers (GFP + and GFP-, each) were evaluated for a same muscle, which was considered as a single experimental unit. CSA values of untransfected fast or slow myofibers of OIL treated rats were significantly lower than those transfected with pT94 in the same muscles and those, either transfected or untransfected, of soleus muscles from 7-NI treated rats matched on body weight (Within-subject ANOVA and Bonferroni post-hoc analysis). (H) Recombinant Grp94 expression affects nNOS immunolocalization in unloaded myofibers of 7-NI-treated rats. Confocal microscopy images after immunofluorescence staining with anti-nNOS pAb (red fluorescence) of A and U solei. Green fluorescence identifies myofibers transfected with Grp94 cDNA (pT94). To see this illustration in color, the reader is referred to the web version of this article at www.liebertpub.com/ars 
Grp94 still counteracted the loss of nNOS immunofluorescence labeling at the sarcolemma of unloaded solei, since inhibition of nNOS activity by means of 7-NI did not hamper the untethering of the enzyme, as demonstrated by confocal microscopy (Fig. 7H).

Conversely, recombinant Grp94 further decreased the percentage of DNPH-positive myofibers compared with untransfected ones (mean and SE $8.87 \pm 2.53 \%$ and $20.28 \pm 3.49 \%$, respectively, evaluated on 954 GFP-positive myofibers from four unloaded solei of 7-NI treated rats; $p=0.04$, Student's t-test).

\section{Discussion}

This study demonstrates that the SR/ER chaperone Grp94 plays a relevant role against myofiber carbonylation and atrophy of unloaded soleus muscle, and mechanistically explains its involvement identifying nNOS as a new interacting partner. The expression of recombinant Grp94 in unloaded muscle fibers affected nNOS subcellular distribution, favoring its localization at the sarcolemma and countering the occurrence of atrophy and oxidative stress.
A

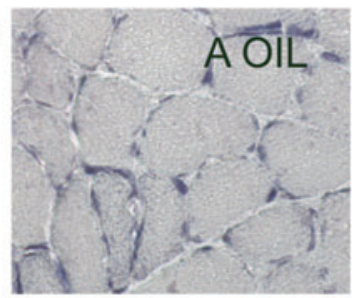

B

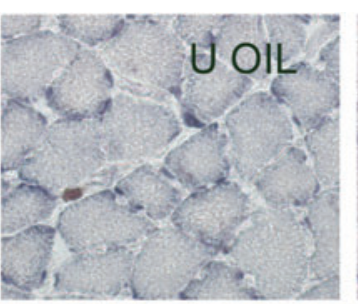

IP: $2 \mathrm{G} 5 \mathrm{mlg}$ $7 \mathrm{NI}$ OIL $7 \mathrm{NI}$

WB nNOS IP: 2 G5 $\mathrm{mlg}$
$7 \mathrm{NI}$ OIL $\mathrm{OIL}$

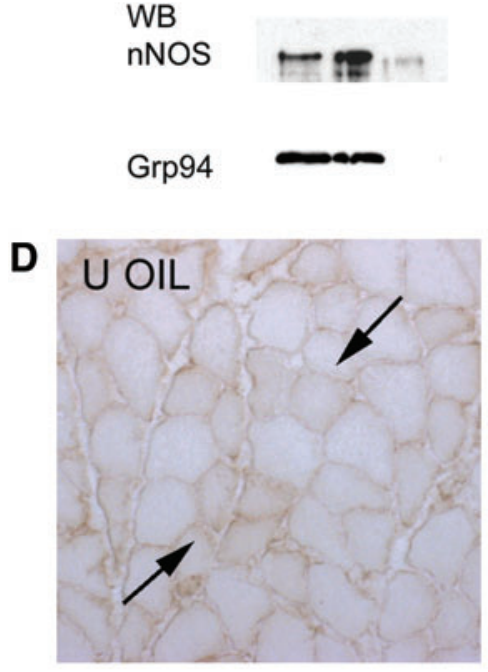

$\mathbf{F}$

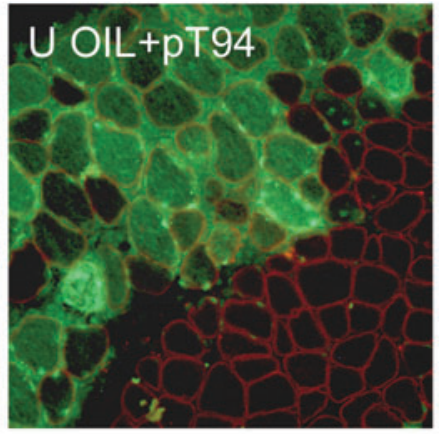

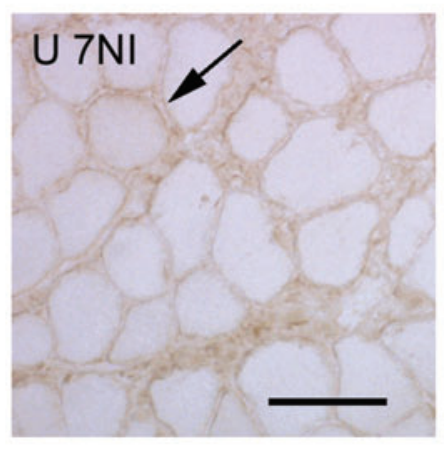

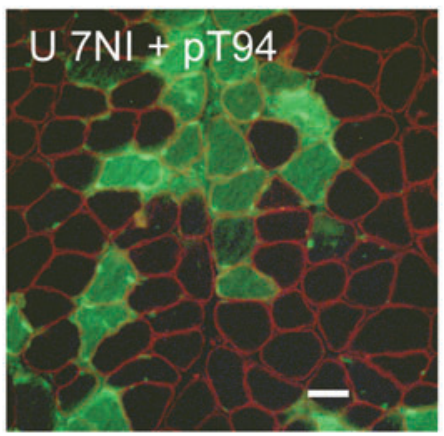

A $7 \mathrm{NI}$
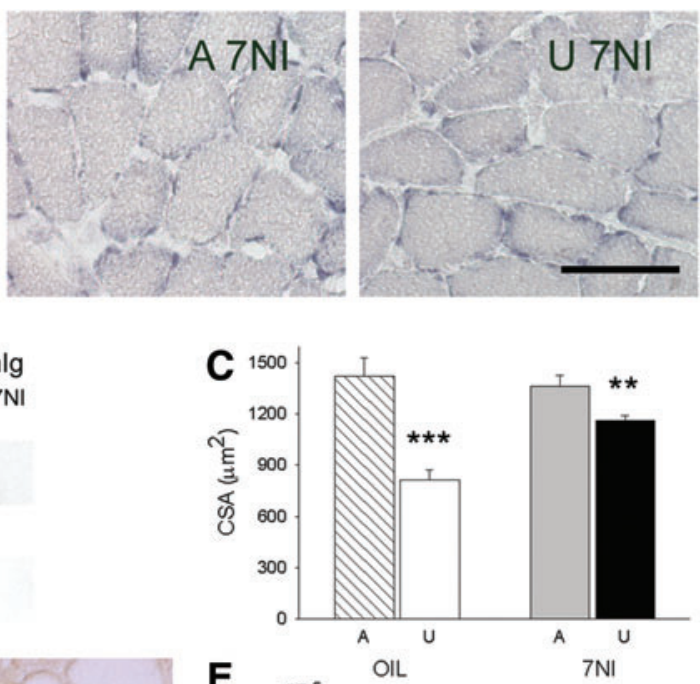

E

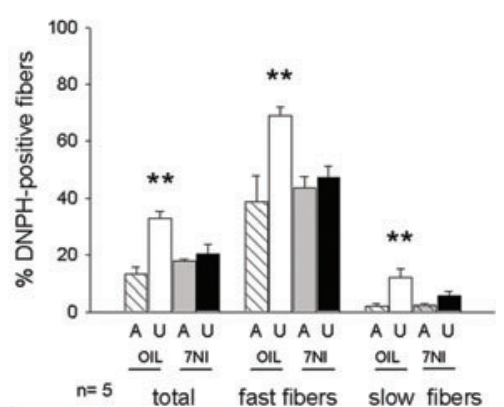

G

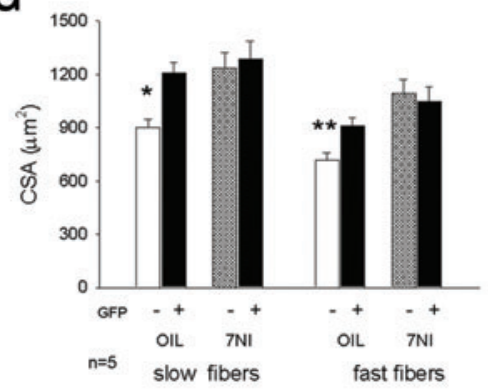

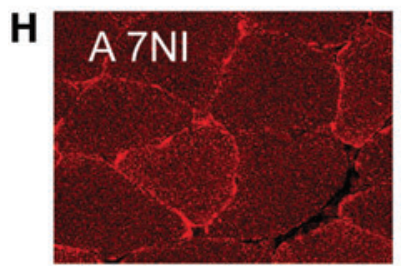
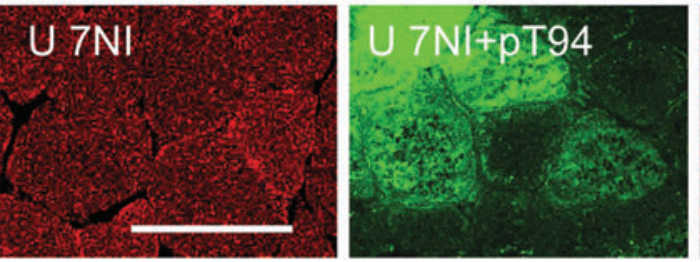

U 7NI+pT94 


\section{Grp94 counteracts atrophy of unloaded soleus muscle}

Grp94 is highly expressed by skeletal myogenic cells, where it is specifically involved in differentiation and maturation $(14,19,44)$. The absolute requirement of this chaperone for IGF folding and secretion hampered muscle cell differentiation in Grp94 knock-out (KO) embryoid bodies (69) and decreased muscle size after conditional muscle-specific Grp94 KO (5). Further, Grp94 possesses calcium-binding ability and participates in the control of calcium homeostasis, displaying cytoprotective properties, which appear to be positively related to the intracellular chaperone protein levels $(4,46,67)$. Different from developing muscles and other adult tissues, Grp94 is expressed at low protein levels in adult mammalian skeletal myofibers $(24,63,65,66)$, and here we show that unweighting decreases them further.

Stoichiometry represents a relevant feature of chaperoneclient protein interaction (39). Increased expression of cytosolic chaperones/stress-proteins, such as Hsp70 and Hsp27, attenuated muscle atrophy secondary to limb immobilization $(12,55)$. Here, we demonstrate that muscle atrophy could be mitigated antagonizing Grp94 decrease by means of the expression of a recombinant one, whereas no effect on myofiber size was observed in standard caged rats. Due to the low number of transfected fibers we could not demonstrate changes in Grp94 protein levels; nevertheless, an indirect proof of the relevance to maintain adequate Grp94 protein amount during unloading was provided by the lack of effects after transfection with two differently deleted Grp94 forms.

A first mechanistic consideration has to take into account the possible involvement of IGFs. Although our findings would not exclude increased IGF maturation by recombinant Grp94, the lack of atrophy attenuation of adjacent untransfected myofibers questions IGFs as the actual mediators of the chaperone effects. Resistance to IGF signaling characterizes exposure to microgravity and hindlimb unloading $(1,20,34,56)$. Further, autocrine and paracrine effects of IGFs against muscle atrophy apparently occur concomitantly, since atrophy attenuation interested both transfected and untransfected myofibers after electroporation with IGF-I cDNA (53). Therefore, our results suggested the requirement of Grp94 for different intrinsic regulatory pathways, whose disruption contributed to muscle disuse atrophy.

\section{Grp94 as a new partner of nNOS}

Skeletal muscle fibers express multiple nNOS isoforms, which differentially localize at the subsarcolemma and Golgi (45). Although the Golgi nNOS isoform was recently identified as a major negative regulator of muscle contractility, decreased nNOS expression at sarcolemma $(51,60)$ was proposed as an upstream event that would foster disuse atrophy, as demonstrated by the NO-mediated increase of activity of the transcription factor FoxO3a and by the mild degree of muscle atrophy induced by hindlimb unloading in nNOS KO mice (60) and in 7-NI treated rats (60 and this article). At the subsarcolemma, nNOS participates in a multiprotein transmembrane apparatus composed by the dystrophin-glycoprotein complex (8), a linker between the extracellular matrix and the intracellular cytoskeleton. Sarcolemma directly connects to T-tubules and nNOS localization at the T-tubule and junctional SR level would be consistent with evidence showing transverse cross-striations after labeling with anti-nNOS antibodies or enzyme histochemistry for NADPH-d (50), decorated subsarcolemmal vesicles by immunoelectronmicroscopy $(9,68)$, localization of nNOS- and dystroglycan complex-interacting proteins like caveolin 3 in the SR (35), and co-immunoprecipitation with the SR calcium channel Ryanodine Receptor (RyR) 1 (52).

Our immunoprecipitation assays revealed Grp94 as a new putative partner of $160 \mathrm{kDa}$ nNOS, which appeared recruited also in non-muscle tissues, like cerebellum. Such a finding suggests that Grp94-nNOS interaction might not be exclusive of the muscle-specific nNOS- $\mu$ isoform (57). The use of a cellpermeant chemical cross-linker to stabilize interactions among proteins further excluded the artifactual nature of this association and confirmed its occurrence in the microsomal fraction. Further, confocal microscopy revealed that both proteins co-localized, albeit partially, mainly at the A-I band boundaries, namely in regions containing T-tubules and junctional SR, and at the sarcolemma. Subcellular localization of $\mathrm{nNOS}$ is apparently driven by the binding to multiprotein complexes of different composition (75). Also the subcellular distribution of Grp94 varies: the protein translocates from ER to Golgi and plasmalemma in differentiating myogenic cells $(14,19)$, and in mammalian adult muscle it accumulates in the junctional SR, since anti-Grp94 antibodies decorated the Z-line in cardiac myocytes (64), and the I band in skeletal myofibers (this article). Proteolysis of intact microsomes suggested that the topology of the interaction between $\mathrm{nNOS}$ (a cytosolic protein) and Grp94 (a SR/ER lumenal one) might be explained by the presence of a minor proportion of Grp94 molecules showing a transmembrane configuration (14, 48 and this article). Alternatively, Grp94 and nNOS might participate to the same multimolecular complex without interacting directly between themselves. Although further studies will be required to identify additional partners, our results show that the expression of recombinant Grp94 was sufficient to hamper the loss of histochemically demonstrated nNOS activity from sarcolemma of unloaded muscle, leading to decreased FoxO-3a nuclear localization and myofiber ubiquitination. Conversely, no effect on subsarcolemmal nNOS localization was observed after the expression of an N-terminal deleted Grp94 protein containing a binding domain for integrins and Toll-like receptors (73). Although the $\Delta 1-570$ Grp94 could not be assayed for chaperone activity, since Grp94 is not required for folding of skeletal muscle integrins (5), it did not apparently bind nNOS, even after chemical cross-linking and despite the presence of some heterodimerization with endogenous Grp94.

\section{Antioxidant effect of recombinant Grp94 expression}

Dysregulation of nNOS activity is a recognized consequence of both decreased enzyme levels and sarcolemmal localization in several types of neuromuscular disorders (13, $17,36,60,74)$. No apparent decrease in protein amount characterized our 7-day unloaded solei, whereas the increased NO availability to the sarcoplasm, indicated by increased cytosolic NADPH-d activity (70 and this article), would not only promote transcription of atrogenes (60), but also hypernitrosylate $\operatorname{RyR}(6,17,52)$, an event that would displace calstabin and lead to calcium leakage from the stores (6). Increased number and frequency of calcium sparks were 
described in the unloaded rat soleus (74), representing a putative trigger of mitochondrial ROS production (11), in addition to NO (33). Oxidative stress of unloaded myofibers was affected by the expression of recombinant Grp94, which reduced sevenfold the percentage of carbonylated myofibers and significantly decreased the presence of active sarcoplasmic nNOS.

In addition to stabilize $\mathrm{nNOS}$ at sarcolemma, recombinant Grp94 might exert antioxidant protection by independently contributing to the maintenance of calcium homeostasis. Overexpression of Grp94 in C2C12 cells reduced the amount of releasable calcium from the stores and prevented protein carbonylation after exposure to hydrogen peroxide (46).

Eventually, the proof of concept demonstration that Grp94 attenuation of disuse-induced myofiber atrophy and carbonylation were mediated by nNOS was obtained by investigating the effects of Grp94 overexpression in unloaded muscles lacking active nNOS, that is, in the presence of significant attenuation of atrophy (60 and this article) and percentage of carbonylated myofibers at ambulatory levels. Expression of recombinant Grp94 did not increase further CSA of unloaded 7-NI-treated solei, whereas it significantly reduced the percentage of carbonylated myofibers to levels comparable to those observed after transfection of both untreated unloaded and ambulatory solei. These results indicate that Grp94 might exert antioxidant cytoprotection through two distinct mechanisms. The first one concerns pathological levels of oxidative stress, like those generated during unloading, and appears mediated by nNOS. The second one concerns oxidative stress levels compatible with physiological muscle activity and its actual effectors remain to be determined.

In conclusion, the findings presented here identify Grp94 as a new partner of nNOS and a major regulator of its localization at the subsarcolemma of unloaded skeletal muscle fibers. Our results also show that changes in protein levels of this ER chaperone/stress protein would occur upstream nNOS untethering from sarcolemma (60). Muscle Grp94 protein levels appeared significantly reduced after few days of mechanical silencing (49). Although such an early decrease remains to be mechanistically investigated, the results presented here reveal the obligatory participation of Grp94 to a multiprotein complex, whose integrity is required to preserve nNOS subcellular localization and ameliorate both trophism and degree of carbonylation of unloaded muscle fibers.

\section{Materials and Methods}

\section{Antibodies}

The following antibodies were used in this study: (i) antiGrp94 antibodies: rabbit polyclonal anti-Grp94 antibody (pAb) (SPA851) and rat anti-Grp94 mAb 9G10, (Stressgen, Victoria, Canada); mouse anti-Grp94 mAbs 3C4 (65) and 2G5 (67); (ii) anti-ER chaperones and proteins: rabbit anti-Grp78 (SPA826) and anti-calreticulin (SPA600), and mouse antiGrp75 mAb (SPS825; Stressgen); goat anti-calnexin pAb (C20; Santa Cruz Biotech., Heidelberg, Germany); mouse anti-Csq $\mathrm{mAb}$ (Affinity BioReagents, Golden, CO); (iii) loading control: mouse anti-GAPDH mAb, clone 6C5 (Millipore, Vimodrone, Italy); (iv) anti-tag antibodies: mouse anti-VSV-G mAb, clone P5D4, conjugated with peroxidase (Sigma, Milan, Italy); rabbit anti-GFP pAb (A11122; Invitrogen, Groningen, The
Netherlands); (v) antibodies for muscle proteins: mouse anti- $\alpha$ sarcoglycan mAb (Monosan, Uden, The Netherlands); antimyosin heavy chain (My) mAbs $(18,61)$, clone BA-D5 for type $1-\beta \mathrm{My}$, clone SC-71 for type 2A My, and clone BF-G6 for $\varepsilon \mathrm{My}$; (vi) anti-nNOS antibodies: rabbit anti-nNOS pAb (H-299; Santa Cruz Biotech.); mouse anti-nNOS mAb clone 16 (BD Transduction Lab., Milan, Italy); (vii) non-immune rabbit and mouse immunogloblulins (rbIg and $\mathrm{mIg}$; Sigma); agaroseconjugated mIg or rbIg (Santa Cruz Biotech.); (viii) goat antiIGF-I and anti-IGF-II pAb (R\&D System, Inc., Lille, France); rabbit anti-FoxO3 (Sigma) and anti-ubiquitin (Dakocytomation, Milano, Italy) pAbs; (xi) secondary antibodies: goat anti$\mathrm{mIg}$ conjugated with horseradish peroxidase or Texas Red and rabbit anti-goat Ig conjugated with horseradish peroxidase (Santa Cruz Biotech.); swine anti-rbIg conjugated with horseradish peroxidase (Dakocytomation); goat anti-rbIg conjugated with Alexa 568 and goat anti-mIg conjugated with Alexa 488 (Invitrogen); anti-rbIg and anti-mIg conjugated with agarose (eBioscience, San Diego, CA).

\section{Plasmids}

The following constructs were used: (i) pT94 (67), a bicistronic vector that contained the cDNA sequences of rabbit Grp94 (clone 2.52) and GFP; (ii) pT, the corresponding empty vector that contained GFP cDNA only (Invitrogen); (iii) pVSV-G5B and pVSV-G6B, which contained the SacII-BamHI fragment and the StyI-BamHI fragment of rabbit Grp94 cDNA, respectively, codifying for $\mathrm{N}$-terminal deleted proteins (AA 272-782 and 571-782, respectively), inserted downstream the sequences codifying for the 21AA of the signal peptide (67) and for a doubled VSV-G tag framed by a PPGP peptide (AYTDIEMQRLGKL-PPGP-AYTDIEMQRLGKL); (iv) $\mathrm{pBK}-\mathrm{RSV}$, the corresponding empty vector (Stratagene, Agilent Techn., Cernusco sul Naviglio, Italy).

\section{Animals and experimental protocols}

A total of 94 six-weeks-old female Wistar rats of about 140-160 g were used for hindlimb unloading associated or not to gene transfer experiments. Number of animal used in each protocol was specified in the text and/or in the following paragraphs.

Hindlimb unloading. The experimental protocol was performed following the recommendations provided by the European Convention for the protection of Vertebrate Animals used for Experimental and Scientific purposes (Council of Europe number 123, Strasbourg, 1985) and authorized by the Animal Ethics Committee of the University of Padova and the Italian Health Ministry (103/2007B).

Animals were caged individually and were randomly assigned to either of two experimental groups $(n=7$ in each group): freely ambulating controls and 7-day hindlimbunloaded animals. Hindlimb muscles were unloaded using the tail-suspension model (10), by wrapping the tail root with tape under general anesthesia induced with intraperitoneal administration of $20 \mathrm{mg} / \mathrm{kg}$ of Zolazepam chlorhydrate and tiletamine chlorhydrate (Zoletil; Virbac SRL, Milano, Italy). Each animal was weighted before and after the suspension period. Animals were euthanized after inducing anesthesia with isofluorane. Soleus muscles were excised, weighed, and frozen in liquid nitrogen. Cerebellum was also excised from control rats. 
In vivo gene transfer. After inducing general anesthesia with isofluorane, soleus muscle was exposed and bilaterally injected with $50 \mu \mathrm{g}$ of purified plasmid. After closing the wound, a train of six 20 ms electrical impulses at $209 \mathrm{~V} / \mathrm{cm}$, with $200 \mathrm{~ms}$ intervals, was given with a BTX Electro Square Porator (Harvard Apparatus, Quebec, Canada) using two $19 \mathrm{~mm}$-squared plate electrodes placed upon the anterior and posterior sides of the hindlimb skin, as described by Serrano et al. (54). The day after, animals were either tail-suspended or let free to ambulate to form different groups of at least seven rats each: (i) freely ambulating animals transfected with the empty vector; (ii) freely ambulating animals transfected with either pT94 or pVSV-G6B or pVSV-G5B; (iii) hindlimb-unloaded animals transfected with the empty vector; (iv) hindlimb-unloaded animals transfected with either pT94 or pVSV-G6B or pVSV-G5B. After 7 days, animals were sacrificed, and soleus muscles processed as described above. Muscles containing less than 20 transfected, nonregenerating, myofibers were excluded from the study.

7-Nitroindazole. The nNOS-specific inhibitor 7-NI was daily administered by intraperitoneal injection at a dosage of $50 \mathrm{mg} / \mathrm{kg}$ to 10 rats following previously described protocols (60). Additional 10 rats were injected with vehicle only. The next day, the left soleus of each animal was electroporated with pT94, whereas the right one was left intact. On the third day after drug or vehicle administration, animals were randomly tail-suspended or let free to ambulate, for 7 days, to form four different groups of five rats each: (i) 7-NI treated freely ambulating animals; (ii) oil-treated freely ambulating animals; (iii) 7-NI treated hindlimb-unloaded animals; and (iv) oil-treated hindlimb-unloaded animals.

\section{Oxyblot and Western blot procedures}

Oxyblot assay was performed as previously described (10), using about $12 \mu \mathrm{g}$ of muscle proteins for derivatization with DNPH (Oxyblot Oxidized Protein Detection Kit; Millipore). The final mix was separated by denaturing gel electrophoresis and proteins transferred to nitrocellulose membranes, which were first stained by Red Ponceau (RP), and then saturated and incubated with anti-DNPH antibody, following manufacturer's instructions. Blots were developed using a chemiluminescence detection system (BioRad Lab., Rome, Italy).

Routine western blot analysis was performed as previously described (61). About $50 \mu \mathrm{g}$ of muscle homogenate, lysed with electrophoresis loading buffer (ELB), containing 3\% sodium dodecyl sulphate (SDS) and 5\% $\beta$-mercaptoethanol, and boiled for $5 \mathrm{~min}$, was loaded on $10 \%$ linear SDS-polyacrylamide gels and electrophoretically separated proteins were electrotransferred onto nitrocellulose. Blots were stained first with RP, saturated, incubated with primary and secondary antibodies conjugated with peroxidase and developed as described above. Densitometry of the reactive polypeptides detected in Oxyblots and western blots was performed on scanned autoradiographic films using the National Institute of Health Image J software, and values were normalized using the corresponding densitometric value of either SA visualized by RP staining (10) or GAPDH immunolabeling.

\section{Immunofluorescence and immunohistochemistry}

Serial consecutive transverse cryosections from soleus muscle were assayed for indirect peroxidase immunohistochemistry and/or immunofluorescence, following previously described protocols (61). In brief, after a $10 \mathrm{~min}$ fixation at room temperature (RT) with $4 \%$ buffered paraformaldehyde (PFA), cryosections were incubated overnight at $4^{\circ} \mathrm{C}$ with the primary antibody, extensively rinsed with phosphate buffered saline (PBS), and incubated with the appropriate secondary antibody for $1 \mathrm{~h}$ at RT.

Incubation with anti-Grp94 antibody required a $10 \mathrm{~min}$ Triton-X100 permeabilization after fixation, as previously described (66).

Cold acetone was used to fix for $10 \mathrm{~min}$ at $-20^{\circ} \mathrm{C}$ cryosections to be incubated with anti-My antibodies (61).

For indirect immunoperoxidase, fixation was followed by inhibition of endogenous peroxidase activity with methanol added with $0.3 \% \mathrm{H}_{2} \mathrm{O}_{2}$ for $30 \mathrm{~min}$ at RT. Bound antibodies were then revealed by incubation with diaminobenzidine.

For control staining, sections were incubated with $1 \mu \mathrm{g} / \mathrm{ml}$ of non-immune $\mathrm{mIg}$ or rbIg as the primary antibody.

Consistency of the immunostaining was validated by independent analysis of adjacent cryosections. Analysis was performed using the Zeiss Microscope Axioplan equipped with fluorescence optics and $10 \times$ and $40 \times$ Plan-Neofluar objectives (Zeiss, Milan, Italy). Images were acquired using a Leica digital DFC 300FX camera and the IM50 software (Leica Microsystems SRL, Milan, Italy). Myofiber CSA was evaluated using Image J software. CSA value for each myofiber population (slow-fast fibers or transfected-untransfected ones) corresponded to the mean of the CSA values measured from 20 to 40 fibers of the same muscle (the experimental unit) and was expressed in $\mu \mathrm{m}^{2}$ (61).

\section{DNPH immunohistochemistry}

Cryosections were reacted with DNPH diluted 1:100 (Oxyblot Oxidized Protein Detection Kit) for $1 \mathrm{~h}$ at RT. After a $10 \mathrm{~min}$ rinse in $50 \mathrm{mM}$ Tris pH7.6 added with $0.9 \% \mathrm{NaCl}$, sections were saturated with $3 \%$ bovine SA dissolved in the same buffer for $20 \mathrm{~min}$, and incubated with the anti$\mathrm{DNPH}$ antibody overnight at $4^{\circ} \mathrm{C}$. After adequate rinses in PBS, sections were incubated for $2 \mathrm{~h}$ at RT with peroxidaseconjugated secondary antibodies and developed with diaminobenzidine.

\section{Cell cultures and in vitro gene transfer}

Cultures of proliferating and 6-h differentiating cells of the murine cell line C2C12 (ECACC) were performed in $10 \mathrm{~cm}$ Petri dishes, as previoulsy described (14). Chemical crosslinking was performed by exposing cells for $5 \mathrm{~min}$ at RT to $330 \mu \mathrm{g} / \mathrm{ml}$ dithiobis-succinimidylpropionate (DSP; Pierce; Euroclone, Pero, Italy) in PBS as previously described (14).

Transient transfection of C2C12 cells with pVSV-G 6B or the empty vector was performed following the procedure described in Pizzo et al. (46). About 10,000 cells/well were seeded in 18 wells of a 24-well plate and used $24 \mathrm{~h}$ after transfection. After extensive rinsing with PBS, cultures were cross-linked and processed for immunoprecipitation.

\section{Immunoprecipitation}

Samples from soleus muscle and cerebellum of ambulating control rats were lysed with $10 \mathrm{mM}$ Tris- $\mathrm{HCl}$ pH 8.0 containing $150 \mathrm{mM} \mathrm{NaCl}, 1 \mathrm{mM}$ ethylenediaminetetraaceticacid 
(EDTA), $50 \mathrm{mM} \mathrm{NaF}$, and $1 \%$ NonIdet-P40, added before use with $1 \mathrm{mM}$ phenylmethylsulfonyl fluoride (PMSF), $10 \mu M$ calpeptin, $1 \mu \mathrm{g} / \mathrm{ml}$ each of leupeptin, pepstatin, and aprotinin and supernatants collected after $30 \mathrm{~min} 10,000 \mathrm{~g}$ centrifugation at $4^{\circ} \mathrm{C}$. Cross-linked cell cultures were lysed with $10 \mathrm{mM}$ Na phosphate, $150 \mathrm{mM} \mathrm{NaCl}, 1 \% \mathrm{NP} 40$, and $0.5 \%$ Na deoxycholate, pH 7.4 added with $1 \mathrm{mM}$ EDTA (RIPA) and antiproteases and supernatants collected after $30 \mathrm{~min} 10,000 \mathrm{~g}$ centrifugation at $4^{\circ} \mathrm{C}$. About $20 \mu \mathrm{g}$ protein from cerebellum lysate, $200 \mu \mathrm{g}$ protein from skeletal muscle, and $150 \mu \mathrm{g}$ from cultures were precleared for $30 \mathrm{~min}$ at $4^{\circ} \mathrm{C}$ in orbital agitation with a mixture of agarose conjugated with either anti-mIg or anti-rbIg. Supernatants were then used for immunoprecipitation with $5 \mu \mathrm{g}$ of primary antibody or non-immune rbIg or $\mathrm{mIg}$ conjugated with agarose. Samples were then incubated with $50 \mu \mathrm{l}$ of the appropriate secondary antibody conjugated with agarose for $1 \mathrm{~h}$ at $4^{\circ} \mathrm{C}$ under agitation. After three rinses with $10 \mathrm{mM}$ Na phosphate and $100 \mathrm{mM} \mathrm{NaCl}, \mathrm{pH} 7.4$ added with antiprotease cocktail, bound proteins were eluted with $35 \mu \mathrm{l} \mathrm{ELB}$, boiled for $5 \mathrm{~min}$, and further analyzed by $7.5 \%$ SDS-polyacrylamide gel electrophoresis and western blotting. A $6-12 \%$ gradient gel electrophoresis was used to separate samples of cultures transfected with the deleted Grp94 form.

\section{Microsome fractionation, digestion, and cross-linking}

Crude membranes were prepared from soleus muscles essentially as described by Margreth et al. (40). Briefly, dissected muscles were minced; homogenized in $10 \mathrm{mM}$ HEPES $\mathrm{pH}$ 7.4, $20 \mathrm{mM} \mathrm{KCl}, 1 \mathrm{mM} \mathrm{PMSF}$, and $10 \mu \mathrm{g} / \mathrm{ml}$ leupeptin; and centrifuged at $650 \mathrm{~g} \times 10 \mathrm{~min}$ at $4^{\circ} \mathrm{C}$. Supernatant was then centrifuged at $10,000 \mathrm{~g} \times 10 \mathrm{~min}$ at $4^{\circ} \mathrm{C}$ to eliminate mitochondria and again at $120,000 \mathrm{~g} \times 90 \mathrm{~min}$ at $4^{\circ} \mathrm{C}$ to yield the total membrane fraction.

Pellet was resuspended with $10 \mathrm{mM}$ Tris $\mathrm{HCl} \mathrm{pH} \mathrm{7.4,} 0.25$ $M$ saccharose, and $1 \mathrm{mM}$ EDTA for PK digestion assays, which were perfomed as previously described (14). For chemical cross-linking, microsomes were resuspended in $0.1 \mathrm{M}$ Na phosphate $\mathrm{pH} 7.2,3 \mathrm{mM} \mathrm{MgCl}$, and $0.1 \mathrm{mM}$ EDTA at a concentration of $2.5 \mathrm{mg} / \mathrm{ml}$. DSP was added to a final concentration of $0.5 \mathrm{mM}$ and incubated for $30 \mathrm{~min}$ on ice. Reaction was stopped by adding Tris $\mathrm{HCl} \mathrm{pH} 7.5$ to a final concentration of $50 \mathrm{mM}$ and lysing membranes with RIPA added with antiproteases.

Crude membranes were prepared from cross-linked C2C12 cell cultures, as described by Kang and Welch (28). After rinsing with PBS, cells were scraped and solubilized with Dounce in $10 \mathrm{mM}$ Tris- $\mathrm{HCl}$ pH 7.4 added with anti-proteases. Lysates were equilibrated with sucrose to $0.25 \mathrm{M}$ and centrifuged at $650 \mathrm{~g} \times 10 \mathrm{~min}$ at $4^{\circ} \mathrm{C}$. Supernatant was centrifuged at $10,000 \mathrm{~g} \times 10 \mathrm{~min}$ at $4^{\circ} \mathrm{C}$ and then at $100,000 \mathrm{~g} \times 90 \mathrm{~min}$ at $4^{\circ} \mathrm{C}$. Pellet was resuspended with RIPA added with antiproteases.

\section{Confocal microscopy}

Transverse and longitudinally oriented cryosections from soleus muscle were treated as described (64) and used either for single immunofluorescence with anti-Grp94 pAb or double immunofluorescence with anti-nNOS pAb and the antiGrp94 mAb 2G5. After adequate rinses with PBS, sections were incubated for $2 \mathrm{~h}$ at RT with a mixture of appropriate goat secondary anti-Ig conjugated with Alexa 568 or 488. Slides were observed with a Leica SP5 confocal microscope equipped with Argon $488 \mathrm{~nm}$ and Helium $543 \mathrm{~nm}$ lasers (Leica Microsystem SRL). Acquisition was performed sequentially, collecting signals between 495 and $540 \mathrm{~nm}$ wavelenghts after excitation with Argon 488 laser, and between 584 and $630 \mathrm{~nm}$ for the Helium 543 one. Images were recorded at $1024 \times 1024$ pixel density using a Kalman four-collection average. A nonconfocal bright field image was also collected from the same field. Images were analyzed using Photoshop software.

\section{NADPH-d histochemistry}

NADPH-d activity was demonstrated by histochemistry following previously described procedures $(22,50)$, with minor modifications. In brief, $12 \mu \mathrm{m}$-thick sections of frozen muscle were cut on a cryostat, collected on slides and fixed for 10-20 min with $2 \%$ PFA. After rinsing with PBS, sections were incubated in a medium containing $50 \mathrm{mM}$ Tris- $\mathrm{HCl} \mathrm{pH} 8.00$, $0.2 \%$ Triton-X-100, $0.5 \mathrm{mM}$ nitroblue tetrazolium chloride (Serva; Crescent Chemical Co., Islandia, NY), and $1 \mathrm{mM}$ $\beta$-NADPH (Sigma) for $1 \mathrm{~h}$ at $37^{\circ} \mathrm{C}$. Reaction was stopped by briefly rinsing with distilled water. Coverslips were mounted with glycerin jelly.

\section{Statistical analyses}

All data were expressed as mean \pm SE.

Statistical analysis between two groups was performed utilizing Student's $t$-test. Paired Student's $t$-test analysis was used when comparing average CSA or OD values between transfected and untransfected myofibers of the same muscles.

ANOVA or within-subject ANOVA, and Bonferroni test for post-hoc analysis were used for multiple comparison. In the case of within-subject ANOVA, average values of transfected and untransfected myofibers were compared with values obtained from transfected muscles obtained from animals of the same body weight (matched participants).

$p$ value $=0.05$ was set as the limit for significance.

All these statistical analyses were performed using SigmaStat software, version 2.0 (Jandel Europe, Germany).

\section{Acknowledgments}

We thank R. Rostellato, M. Andreose, and B. Ravara for technical assistance. The financial support of Agenzia Spaziale Italiana (grant OSMA-WP1B51-2 to L.G.) is gratefully acknowledged.

\section{Author Disclosure Statement}

No competing financial interests exist.

\section{References}

1. Allen DL, Bandstra ER, Harrison BC, Thorng S, Stodieck LS, Kostenuik PJ, Morony S, Lacey DL, Hammond TG, Leinwand LL, Argraves WS, Bateman TA, and Barth JL. Effects of spaceflight on murine skeletal muscle gene expression. J Appl Physiol 106: 582-595, 2009.

2. Arbogast S, Smith J, Matuszczak Y, Hardin BJ, Moylan JS, Smith JD, Ware J, Kennedy AR, and Reid MB. Bowman-Birk inhibitor concentrate prevents atrophy, weakness, and oxidative stress in soleus muscle of hindlimb-unloaded mice. J Appl Physiol 102: 956-964, 2007. 
3. Argon Y and Simen BB. Grp94, an ER chaperone with protein and peptide binding properties. Semin Cell Dev Biol 10: 495-505, 1999.

4. Bando Y, Katayama T, Aleshin AN, Manabe T, and Tohyama M. GRP94 reduces cell death in SH-SY5Y cells perturbated calcium homeostasis. Apoptosis 9: 501-508, 2004.

5. Barton ER, Park SH, James JK, Makarewich CA, Philippou A, Eletto D, Lei H, Brisson B, Ostrovsky O, Li Z, and Argon Y. Deletion of muscle GRP94 impairs both muscle and body growth by inhibiting local IGF production. FASEB J 26: 3691-3792, 2012.

6. Bellinger AM, Reiken S, Carlson C, Mongillo M, Liu X, Rothman L, Matecki S, Lacampagne A, and Marks AR. Hypernitrosylated ryanodine receptor calcium release channels are leaky in dystrophic muscle. Nat Med 15: 325-330, 2009.

7. Biswas C, Ostrovsky O, Makarewich CA, Wanderling S, Gidalevitz T, and Argon Y. The peptide-binding activity of GRP94 is regulated by calcium. Biochem J 405: 233-241, 2007.

8. Brenman JE, Chao DS, Xia H, Aldape K, and Bredt DS. Nitric oxide synthase complexed with dystrophin and absent from skeletal muscle sarcolemma in Duchenne muscular dystrophy. Cell 82: 743-752, 1995.

9. Buchwalow IB, Minin EA, Samoilova VE, Boecker W, Wellner M, Schmitz W, Neumann J, and Punkt K. Compartimentalization of $\mathrm{NO}$ signaling cascade in skeletal muscles. Biochem Biophys Res Commun 330: 615-621, 2005.

10. Dalla Libera L, Ravara B, Gobbo V, Tarricone E, Vitadello M, Biolo G, Vescovo G, and Gorza L. A transient anti-oxidant stress-response accompanies the onset of disuse atrophy in human skeletal muscle. J Appl Physiol 107: 549-557, 2009.

11. Davidson SM and Duchen MR. Calcium microdomains and oxidative stress. Cell Calcium 40: 561-574, 2006.

12. Dodd SL, Hain B, Senf SM, and Judge AR. Hsp27 inhibits IKK $\beta$-induced NF-kB activity and skeletal muscle atrophy. FASEB J 23: 3415-3423, 2009.

13. Finanger Hedderick EL, Simmers JL, Soleimani A, AndresMateos E, Marx R, Files DC, King L, Crawford TO, Corse $\mathrm{AM}$, and Cohn RD. Loss of sarcolemmal nNOS is common in acquired and inherited neuromuscular disorders. Neurology 76: 960-967, 2011.

14. Frasson M, Vitadello M, Brunati AM, La Rocca N, Tibaldi E, Pinna LA, Gorza L, and Donella-Deana A. Grp94 is Tyrphosphorylated by Fyn in the lumen of the endoplasmic reticulum and translocates to Golgi in differentiating myoblasts. Biochim Biophys Acta 1793: 239-252, 2009.

15. Fraysse B, Desaphy JF, Pierno S, De Luca A, Liantonio A, Mitolo CI, and Camerino DC. Decrease in resting calcium and calcium entry associated with slow-to-fast transition in unloaded rat soleus muscle. FASEB J 17: 1916-1918, 2003.

16. Furukawa-Hibi Y, Yoshida-Araki K, Ohta T, Ikeda K, and Motoyama N. FOXO forkhead transcription factors induce G(2)-M checkpoint in response to oxidative stress. J Biol Chem 277: 26729-26732, 2002.

17. Gentil C, Leturcq F, Ben Yaou R, Kaplan JC, Laforet P, Pénisson-Besnier I, Espil-Taris C, Voit T, Garcia L, and PiétriRouxel F. Variable phenotype of del45-55 Becker patients correlated to $\mathrm{nNOS} \mu$ mislocalization and RYR1 hypernitrosylation. Hum Mol Genet 21: 3449-3460, 2012.

18. Gorza L, Saggin L, Sartore S, and Ausoni S. An embryonic-like myosin heavy chain is transiently expressed in nodal conduction tissue of the rat heart. J Mol Cell Cardiol 20: 931-941, 1988.

19. Gorza L and Vitadello M. Reduced amount of the glucoseregulated protein GRP94 in skeletal myoblasts results in loss of fusion competence. FASEB J 14: 461-475, 2000.
20. Han B, Zhu MJ, Ma C, and Du M. Rat hindlimb unloading down-regulates insulin like growth factor-1 signaling and AMP-activated protein kinase, and leads to severe atrophy of the soleus muscle. Appl Physiol Nutr Metab 32: 1115-1123, 2007.

21. Hofer T, Marzetti E, Xu J, Seo AY, Gulec S, Knutson MD, Leeuwenburgh $\mathrm{C}$, and Dupont-Versteegden EE. Increased iron content and RNA oxidative damage in skeletal muscle with aging and disuse atrophy. Exp Gerontol 43: 563-570, 2008.

22. Hope BT, Michael GJ, Knigge KM, and Vincent SR. Neuronal NADPH diaphorase is a nitric oxide synthase. Proc Natl Acad Sci U S A 88: 2811-2814, 1991.

23. Hunter RB, Mitchell-Felton H, Essig DA, and Kandarian SC. Expression of endoplasmic reticulum stress proteins during skeletal muscle disuse atrophy. Am J Physiol Cell Physiol 281: C1285-C1290, 2001.

24. Ikezoe K, Furuya H, Ohyagi Y, Osoegawa M, Nishino I, Nonaka I, and Kira J. Dysferlin expression in tubular aggregates: their possible relationship to endoplasmic reticulum stress. Acta Neurophathol 105: 603-609, 2003.

25. Ingalls $\mathrm{CP}$, Warren GL, and Armstrong RB. Intracellular $\mathrm{Ca}^{2+}$ transients in mouse soleus muscle after hindlimb unloading and reloading. J Appl Physiol 187: 386-390, 1999.

26. Jaspers SR and Tischler M. Atrophy and growth failure of rat hindlimb muscle in tail-cast suspension. J Appl Physiol 57: 1472-1479, 1984

27. Kalmar B and Greensmith L. Induction of heat shock proteins for protection against oxidative stress. Adv Drug Deliv Rev 61: 310-318, 2009.

28. Kang HS and Welch WJ. Charactrization and purification of the $94 \mathrm{kDa}$ glucose-regulated protein. J Biol Chem 266: 5643$5649,1991$.

29. Kaul SC, Deocaris CC, and Wadhwa R. Three faces of mortalin: a housekeeper, guardian and killer. Exp Gerontol 42: 263-274, 2007.

30. Koch G, Smith M, Macer D, Webster P, and Mortara R. Endoplasmic reticulum contains a common, abundant calcium-binding glycoprotein, endoplasmin. J Cell Sci 86: 217232, 1986.

31. Kondo H, Miura M, and Itokawa Y. Oxidative stress in skeletal muscle atrophied by immobilization. Acta Physiol Scand 142: 527-528, 1991.

32. Kondo H, Miura M, Nagakaki I, Sasaki S, and Itokawa Y. Trace element movement and oxidative stress in skeletal muscle atrophied by immobilization. Am J Physiol Endocrinol Metab 25: 583-590, 1992.

33. Jacobson J, Duchen MR, Hothersall J, Clark JB, and Heales SJR. Induction of mitochondrial oxidative stress in astrocytes by nitric oxide precedes disruption of energy metabolism. J Neurochem 95: 388-395, 2005.

34. Lalani R, Bhasin S, Byhower F, Tarnuzzer R, and Grant M. Myostatin and insulin-like growth factor-I and -II expression in the muscle of rats exposed to the microgravity environment of the NeuroLab space shuttle flight. J Endocrinol 167: 417-428, 2000.

35. Li C, Duan W, Yang F, and Zhang X. Caveolin-3-anchored microdomains at the rabbit sarcoplasmic reticulum membranes. Biochem Biophys Res Commun 344: 1135-1140, 2006.

36. Li D, Yue Y, Lai Y, Hakim CH, and Duan D. Nitrosative stress elicited by $\mathrm{nNOS} \mu$ delocalization inhibits muscle force in dystrophin-null mice. J Pathol 223: 88-98, 2011.

37. Li Y-P, Chen Y, Li AS, and Reid MB. Hydrogen peroxide stimulates ubiquitin-conjugating activity and expression of genes for specific E2 and E3 proteins in skeletal muscle myotubes. Am J Physiol 285: C806-C812, 2003. 
38. Liu H, Miller E, van de Water B, and Stevens JL. Endoplasmic reticulum stress proteins block oxidant-induced $\mathrm{Ca}^{2+}$ increases and cell death. J Biol Chem 273: 12858-12872, 1998.

39. Marciniak SJ and Ron D. Endoplasmic reticulum stress signaling in disease. Physiol Rev 86: 1133-1149, 2009.

40. Margreth A, Damiani E, and Tobaldin G. Ratio of dihydropyridine to ryanodine receptors in mammalian and frog twitch muscles in relation to the mechanical hypothesis of excitation-contraction coupling. Biochem Biophys Res Commun 197: 1303-1311, 1993.

41. McClung JM, Van Gammeren D, Whidden MA, Falk DJ, Kavazis AN, Hudson MB, Gayan-Ramirez G, Decramer M, DeRuisseau KC, and Powers SK. Apocynin attenuates diaphragm oxidative stress and protease activation during prolonged mechanical ventilation. Crit Care Med 37: 1373-1379, 2009.

42. Min K, Smuder AJ, Kwon O, Kavazis AN, Szeto HH, and Powers SK. Mitochondrial-targeted antioxidants protect skeletal atrophy muscle against immobilization-induced muscle. J Appl Physiol 111: 1459-1466, 2011.

43. Musarò A, Fulle S, and Fanò G. Oxidative stress and muscle homeostasis. Curr Opin Clin Nutr Metab Care 13: 236-242, 2010.

44. Ostrovsky O, Eletto D, Makarewich C, Barton ER, and Argon Y. Glucose regulated protein 94 is required for muscle differentiation through its control of the autocrine production of insulin-like growth factors. Biochim Biophys Acta 1803: 333-341, 2010.

45. Percival JM, Anderson KNE, Huang P, Adams ME, and Froehner SC. Golgi and sarcolemmal neuronal NOS differentially regulate contraction-induced fatigue and vasoconstriction in exercising mouse skeletal muscle. J Clin Invest 120: 816-826, 2010.

46. Pizzo P, Scapin C, Vitadello M, Florean C, and Gorza L. Grp94 acts as a mediator of curcumin-induced anti-oxidant defence in myogenic cells. J Cell Mol Med 14: 970-981, 2010.

47. Powers SK, Smuder AJ, and Criswell DS. Mechanistic links between oxidative stress and disuse muscle atrophy. Antioxid Redox Signal 15: 2519-2528, 2011.

48. Reddy RK, Lu J, and Lee AS. The endoplasmic reticulum chaperone glycoprotein GRP94 with $\mathrm{Ca}(2+)$-binding and antiapoptotic properties is a novel proteolytic target of calpain during etoposide-induced apoptosis. J Biol Chem 274: 28476-28483, 1999.

49. Renaud G, Llano-Diez M, Ravara B, Gorza L, Feng HZ, Jin JP, Cacciani N, Gustafson AM, Ochala J, Corpeno R, Li M, Hedström Y, Ford GC, Nair KS, and Larsson L. Sparing of muscle mass and function by passive loading in an experimental intensive care unit model. J Physiol 591: 1385-1402, 2013.

50. Rothe F, Langnaese K, and Wolf G. New aspects of the location of neuronal nitric oxide synthase in the skeletal muscle: a light and electron microscopic study. Nitric Oxide 13: 21-35, 2005.

51. Rudnick J, Püttmann B, Tesch PA, Alkner B, Schoser BG, Salanova M, Kirsch K, Gunga HC, Schiffl G, Lück G, and Blottner D. Differential expression of nitric oxide synthases (NOS 1-3) in human skeletal muscle following exercise countermeasure during 12 weeks of bed rest. FASEB J 218: 1228-1230, 2004.

52. Salanova M, Schiz G, Rittweger J, Felsenberg D, and Blottner D. Ryanodine receptor type-1 (RyR1) expression and protein S-nitrosylation pattern in human soleus myofibres following bed rest and exercise countermeasure Histochem Cell Biol 130: 105-118, 2008.

53. Schakman $\mathrm{O}$, Gilson $\mathrm{H}$, de Coninck V, Lause P, Verniers J, Havaux X, Ketelslegers JM, and Thissen JP. Insulin-like growth factor-I gene transfer by electroporation prevents skeletal muscle atrophy in glucocorticoid-treated rats. Endocrinology 146: 1789-1797, 2005.

54. Serrano AL, Murgia M, Pallafacchina G, Calabria E, Coniglio P, Lomo T, and Schiaffino S. Calcineurin controls nerve activitydependent specification of slow skeletal muscle fibers but not muscle growth. Proc Natl Acad Sci U S A 98: 13108-13113, 2001.

55. Senf SM, Dodd SL, McClung JM, and Judge AR. Hsp70 overexpression inhibits NF-kB and Foxo3a transcriptional activities and prevents skeletal muscle atrophy. FASEB J 22: 3836-3845, 2008.

56. Shen R, Asa S, Ezzat S, and Gonzalez-Cadavid NF. Rat skeletal muscles atrophized by microgravity exposure showed decreased IGF-II transcript levels and human muscle exposed to simulated microgravity (bed rest) displays early appearance of insulin resistance. J Endocrinol 167: 417-428, 2000.

57. Silvagno F, Xia H, and Bredt DS. Neuronal nitric-oxide synthase- $\mu$, an alternatively spliced isoform expressed in differentiated skeletal muscle. J Biol Chem 271: 11204-11208, 1996.

58. Soldano KL, Jivan A, Nicchitta CV, and Gewirth DT. Structure of the N-terminal domain of GRP94. Basis for ligand specificity and regulation. J Biol Chem 278: 48330-48338, 2003.

59. Stevenson EJ, Giresi PG, Koncarevic A, and Kandarian SC. Global analysis of gene expression patterns during disuse atrophy in rat skeletal muscle. J Physiol 551: 33-48, 2003.

60. Suzuki N, Motohashi N, Uezumi A, Fukada S, Yoshimura T, Itoyama Y, Aoki M, Miyagoe-Suzuki Y, and Takeda S. NO production results in suspension induced muscle atrophy through dislocation of neuronal NOS. J Clin Invest 117: 24682476, 2007.

61. Tarricone E, Scapin C, Vitadello M, Esposito F, Margonato V, Milano G, Samaja M, and Gorza L. Cellular distribution of Hsp70 expression in rat skeletal muscles. Effects of moderate exercise training and chronic hypoxia. Cell Stress Chaper 13: 483-495, 2008.

62. Venojärvi M, Kvist M, Jozsa L, Kalimo H, Hänninen O, and Atalay M. Skeletal muscle HSP expression in response to immobilization and remobilization. Int J Sports Med 28: 281286, 2007.

63. Vinciguerra M, Musaro A, and Rosenthal N. Regulation of muscle atrophy in aging and disease. Adv Exp Med Biol 694: 211-233, 2010.

64. Vitadello M, Ausma J, Borgers M, Gambino A, Casarotto DC, and Gorza L. Increased myocardial GRP94 amounts during sustained atrial fibrillation: a protective response? Circulation 103: 2201-2206, 2001.

65. Vitadello M, Colpo P, and Gorza L. Rabbit cardiac and skeletal myocytes differ in costitutive and inducible expression of the glucose-regulated protein GRP94. Biochem J 332: 351-359, 1998.

66. Vitadello M, Doria A, Tarricone E, Ghirardello A, and Gorza L. Myofiber stress-response in myositis: parallel investigations on patients and experimental animal models of muscle regeneration and systemic inflammation. Arthritis Res Ther 12: R52.

67. Vitadello M, Penzo D, Petronilli V, Michieli G, Gomirato S, Menabò R, Di Lisa F, and Gorza L. Overexpression of the stress-protein Grp94 reduces cardiomyocyte necrosis due to calcium overload and simulated ischemia. FASEB J 17: 923-925.

68. Wakayama $\mathrm{Y}$, Inoue $\mathrm{M}$, Murahashi M, Shibuya S, Jimi T, Kojima $\mathrm{H}$, and Oniki H. Ultrastructural localization of alpha 1-syntrophin and neuronal nitric oxide synthase in normal skeletal myofiber, and their relation to each other and to dystrophin. Acta Neuropathol 94: 455-464, 1997. 
69. Wanderling S, Simen BB, Ostrovsky O, Ahmed NT, Vogen SM, Gidalevitz T, and Argon Y. GRP94 is essential for mesoderm induction and muscle development because it regulates insulinlike growth factor secretion. Mol Biol Cell 18: 3764-3775, 2007.

70. Wei IH, Tu HC, Huang CC, Tsai MH, Tseng CY, and Shieh JY. 2011. Epigallocatechin gallate attenuates NADPH-d/ nNOS expression in motor neurons of rats following peripheral nerve injury. BMC Neurosci 12: 52, 2011.

71. Weiss N, Andrianjafiniony T, Dupré-Aucouturier S, Pouvreau $\mathrm{S}$, Desplanches D, and Jacquemond V. Altered myoplasmic $\mathrm{Ca} 2+$ handling in rat fast-twitch skeletal muscle fibres during disuse atrophy. Pflugers Arch Eur J Physiol 459: 631-644, 2010.

72. Whidden MA, McClung JM, Falk DJ, Hudson MB, Smuder AJ, Nelson WB, and Powers SK. Xanthine oxidase contributes to mechanical ventilation-induced diaphragmatic oxidative stress and contractile dysfunction. J Appl Physiol 106: 385-394, 2010.

73. Wu S, Hong F, Gewirth D, Guo B, Liu B, and Li Z. The molecular chaperone gp96/Grp94 interacts with toll-like receptors and integrins via its c-terminal hydrophobic domain. J Biol Chem 287: 6735-6742, 2012.

74. Xu PT, Li Q, Sheng J, Chang H, Song Z, and Yu ZB. Passive stretch reduces calpain activity through nitric oxide pathway in unloaded soleus muscles. Mol Cell Biochem 367: 113-124, 2012.

75. Zhou L and Zhu DY. Neuronal nitric oxide synthase: structure, subcellular localization, regulation, and clinical implications. Nitric Oxide 20: 223-230, 2009.

Address correspondence to: Prof. Luisa Gorza

Department of Biomedical Sciences

University of Padova

Viale G. Colombo 3

Padova 35121

Italy

E-mail: luisa.gorza@unipd.it

Date of first submission to ARS Central, July 3, 2012; date of final revised submission, September 24, 2013; date of acceptance, October 5, 2013.

$\begin{aligned} & \text { Abbreviations Used } \\ 7-\mathrm{NI} & =7 \text {-nitroindazole } \\ \mathrm{amb} \text { or } \mathrm{A}= & \text { ambulating } \\ \mathrm{ANOVA} & =\text { analysis of variance } \\ \mathrm{CE} & =\text { cerebellum }\end{aligned}$

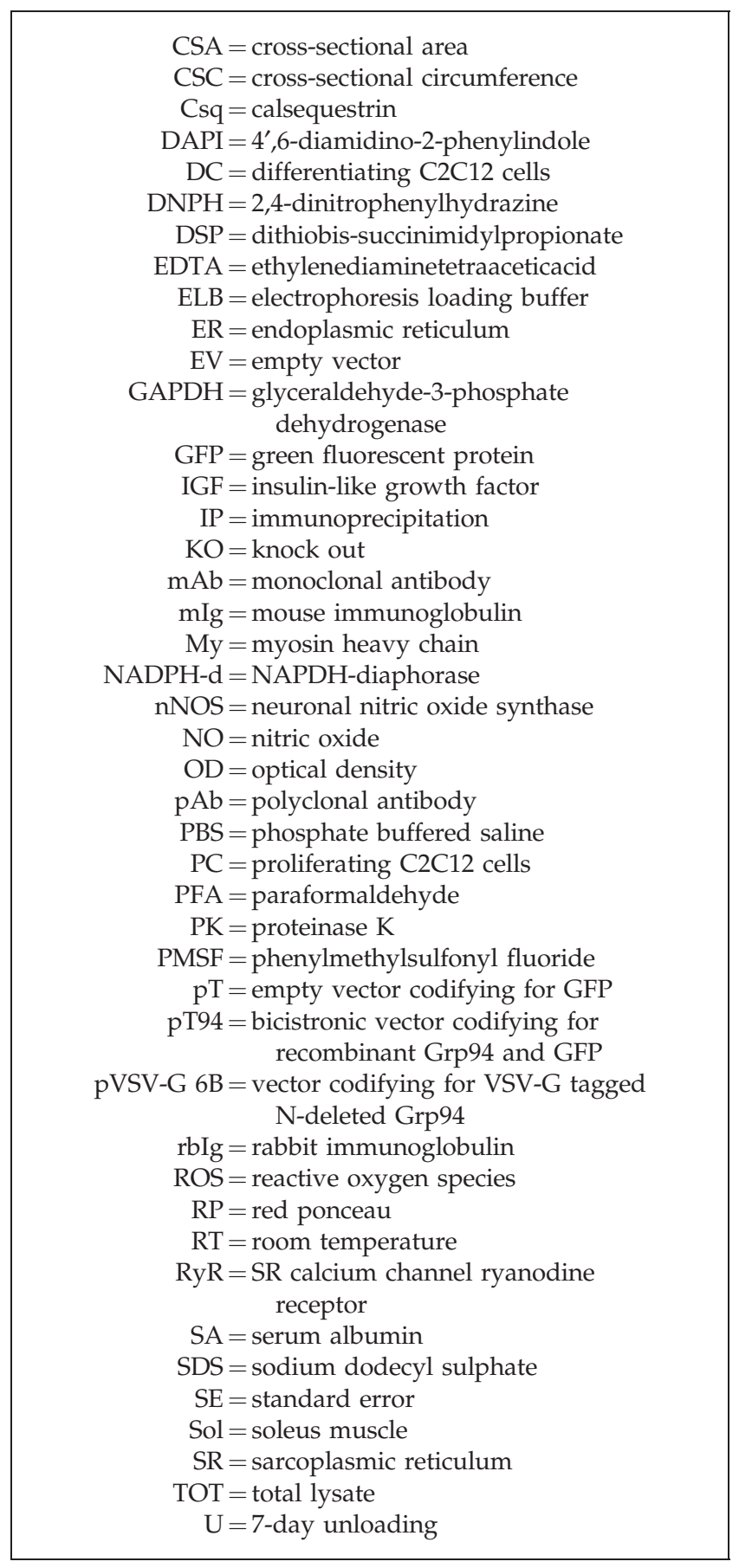

